\title{
A GPCR-based yeast biosensor for biomedical, biotechnological, and point-of-use cannabinoid determination
}

\section{Karel Miettinen}

University of Copenhagen

\section{Nattawat Leelahakorn}

University of Copenhagen

\section{Aldo Almeida}

University of Copenhagen https://orcid.org/0000-0001-5284-8992

\section{Yong Zhao}

University of Copenhagen

\section{Lukas Hansen}

University of Copenhagen

Iben Nikolajsen

University of Copenhagen

Jens Andersen

University of Copenhagen https://orcid.org/0000-0003-1720-1581

Michael Givskov

University of Copenhagen

\section{Dan Stærk}

University of Copenhagen https://orcid.org/0000-0003-0074-298X

\section{Søren Bak}

University of Copenhagen https://orcid.org/0000-0003-4100-115X

Sotirios Kampranis ( $\nabla$ soka@plen.ku.dk)

University of Copenhagen

\section{Article}

Keywords:

Posted Date: January 13th, 2022

DOI: https://doi.org/10.21203/rs.3.rs-743436/v1 
License: (c) (i) This work is licensed under a Creative Commons Attribution 4.0 International License. Read Full License

Version of Record: A version of this preprint was published at Nature Communications on June 27th, 2022. See the published version at https://doi.org/10.1038/s41467-022-31357-6. 


\section{A GPCR-based yeast biosensor for biomedical, biotechnological,}

\section{and point-of-use cannabinoid determination}

Karel Miettinen ${ }^{1}$, Nattawat Leelahakorn ${ }^{1}$, Aldo Almeida ${ }^{1,2}$, Yong Zhao ${ }^{1}$, Lukas Ronild Hansen ${ }^{1}$, Iben Egebæk Nikolajsen ${ }^{1}$, Jens B. Andersen ${ }^{3}$, Michael Givskov${ }^{3}$, Dan Staerk ${ }^{4}$, Søren Bak ${ }^{1}$, and Sotirios C. Kampranis ${ }^{1, *}$

${ }^{1}$ Biochemical Engineering Group, Plant Biochemistry Section, Department of Plant and Environmental Sciences, University of Copenhagen, Thorvaldsensvej 40, 1871 Frederiksberg C, Denmark.

${ }^{2}$ Bioremediation Laboratory, Faculty of Biological Sciences, Autonomous University of Coahuila, Carretera Torreón-Matamoros km. 7.5, Torreón, Coahuila, 27000, Mexico

${ }^{3}$ Department of Immunology and Microbiology, Faculty of Health and Medical Sciences, University of Copenhagen, Blegdamsvej 3B, 2200 Copenhagen, Denmark

${ }^{4}$ Department of Drug Design and Pharmacology, Faculty of Health and Medical Sciences, University of Copenhagen, Universitetsparken 2, 2100 Copenhagen, Denmark

${ }^{*}$ Corresponding author: Email: soka@plen.ku.dk 


\section{ABSTRACT}

The decriminalization of cannabis and the growing interest in cannabinoids as therapeutics require efficient methods to discover novel compounds and monitor cannabinoid levels in human samples and products. However, current methods are limited by the structural diversity of the active compounds. Here, we construct a G-protein coupled receptor-based yeast whole-cell biosensor, optimize it to achieve high sensitivity and dynamic range, and prove its effectiveness in three real-life applications. First, we screen a library of compounds to discover two novel agonists and two antagonists and demonstrate that our biosensor can democratize GPCR drug discovery by enabling low-cost highthroughput analysis using open-source automation. Subsequently, we bioprospect 51 plants to discover a novel phytocannabinoid, dugesialactone. Finally, we develop a robust portable device, analyze bodyfluid samples, and confidently detect illicit synthetic drugs like "Spice"/“K2". Taking advantage of the extensive sensing repertoire of GPCRs, this technology can be extended to detect numerous other compounds. 149 
1 G-protein coupled receptors (GPCRs) are the main sensing entities of higher eukaryotes. They confer

2 the ability to see, smell, and taste, and play key roles in endocrine signaling and the regulation of the

3 immune system ${ }^{1}$. As such, GPCRs have evolved to detect molecules with tremendous chemical

4 diversity, from small compounds to peptides. Harnessing the sensing capability of GPCRs can have

5 profound applications for biotechnology, enabling specific detection of an immense diversity of ligands.

6 Whole-cell biosensors based on microbial cells, such as Saccharomyces cerevisiae, armed with GPCRs

7 as sensing entities can provide robust detection of different molecules with considerable advantages in

8 terms of cost, diversity and portability. Work in this direction has established a general framework for

9 integrating heterologous GPCR signaling in yeast ${ }^{2-5}$ However, achieving the sensitivity, throughput, and

10 ease-of-use that is essential for most biotechnological applications requires further development. Here,

11 we choose a key application, determination of cannabinoid compounds, to further develop this

12 technology and showcase its performance in challenging, real-life, problems.

14 Cannabinoids, the bioactive compounds of cannabis plants, have potent analgesic and anti15 inflammatory properties and have been used in traditional medicine for millennia ${ }^{6}$. However, in the early 16 20th century, cannabis was made illegal due to its psychoactive effects, and, as a result, cannabinoids 17 have been largely neglected by modern medicine. Recently, a strong interest in cannabinoids has re18 emerged as several studies have demonstrated that cannabinoids have the potential to delay the 19 progression of neurodegenerative diseases such as Alzheimer's, Huntington's, and multiple sclerosis ${ }^{7,8}$. 20 This interest is reflected in more than 500 currently ongoing clinical trials involving cannabis or 21 cannabinoids worldwide (clinicaltrials.gov). In combination with the decriminalization of the use of cannabis for recreational and medicinal use, the demand for cannabis and its products has sharply risen and the world market for cannabinoid-based pharmaceuticals is expected to reach $\$ 25$ billion by $2025^{9}$.

25 Cannabinoids exert their activity in humans by targeting the canonical cannabinoid receptors $\mathrm{CB} 1^{10}$ and CB2 ${ }^{11}$. In addition to (-)-trans- $\Delta^{9}$-tetrahydrocannabinol (THC), cannabidiol (CBD), and the other structurally related molecules found in cannabis plants, the cannabinoid receptors are also targeted by 
several structurally non-related types of compounds. This diverse group of ligands encompasses endocannabinoids (the endocrine signaling molecules naturally synthesized by humans and other animals ${ }^{12,13}$ ), other structurally-distinct plant natural products (termed collectively as phytocannabinoids ${ }^{14}$ ), and different types of synthetic compounds (many of which are used as illicit drugs $)^{15,16}$. For simplicity, herein, we use the term cannabinoids collectively to refer to any ligand of CB1 and $\mathrm{CB} 2$, regardless of structure or origin. Both $\mathrm{CB} 1$ and $\mathrm{CB} 2$ are GPCRs. CB1 is present in the central nervous system and modulates neurotransmitter release ${ }^{13}$, whereas CB2 plays a role in the immune system $^{17-21}$.

The rapidly rising interest in cannabinoids has resulted in a sharp increase in demand for canonical cannabinoids but also for novel natural or synthetic compounds specifically targeting CB2. Developing easy-to-use, cost-effective, and high-throughput tools will permit the rapid initial screening of the constantly expanding in size compound libraries produced by combinatorial chemistry (Fig. 1A). Furthermore, the discovery of novel drug leads from plant extracts or microorganisms requires assays with high sensitivity and selectivity, as these compounds are often present in minute amounts in highly complex mixtures (Fig. 1A). In addition, the decriminalization of the use of cannabis for self-medication and recreation in several countries creates a need for facile methods to determine the potency of commercial cannabis preparations and regulate its public use. Moreover, the increasing number of supervised therapeutic approaches that employ cannabinoids requires regular monitoring of cannabinoid levels in body fluids during the course of treatment. Importantly, there is an urgent need to analyze the presence of illicit synthetic compounds, such as "Spice" or "K2", which have been associated with several cases of poisoning ${ }^{16,22,23}$. Many of these challenges require the development of convenient and robust methods so that non-trained personnel outside laboratory settings can detect the presence of cannabinoids in real-life samples such as those obtained from urine or saliva (Fig. 1A).

The broad chemical diversity of compounds that modulate the cannabinoid receptors poses a considerable challenge on the development of a single method that can efficiently and reliably address 
55 all the above needs. Here, we propose that the solution to this is a yeast whole-cell biosensor that uses the CB2 receptor to detect the whole range of its structurally diverse ligands. Baker's yeast has been shown to be suitable for the functional expression of several GPCRs, including the CB1 and CB2 receptors $^{2,4,5}$. This is possible because GPCR signaling pathways, such as the yeast pheromone pathway, share a highly conserved architecture consisting of analogous components between kingdoms. As a general mechanism, upon ligand binding, each GPCR receptor activates a dedicated Ga protein that, in turn, dissociates from the heterotrimeric Gaßy complex. In the yeast pheromone pathway, the resulting Gßy dimer triggers a MAPK cascade, which in turn activates a transcription factor (Ste12p) that finally drives the expression of pathway response genes. In previous work, it has been possible to hijack the yeast pheromone pathway by replacing the pheromone receptor with a GPCR of interest and monitor the pathway's downstream response using a reporter gene ${ }^{4,5,24-26}$. This has been broadly exploited for example, in the study of specific receptors ${ }^{27,28}$, deorphanization of uncharacterized receptors $^{25}$, study of cell-cell communication ${ }^{29}$ guiding metabolic engineering efforts ${ }^{30}$ and detection of fungal pathogens ${ }^{24}$. However, specialized yeast biosensors capable of performing low-cost highthroughput bioactivity characterization, bioprospecting, and, especially, out-of-lab applications, have yet to be introduced. 
A

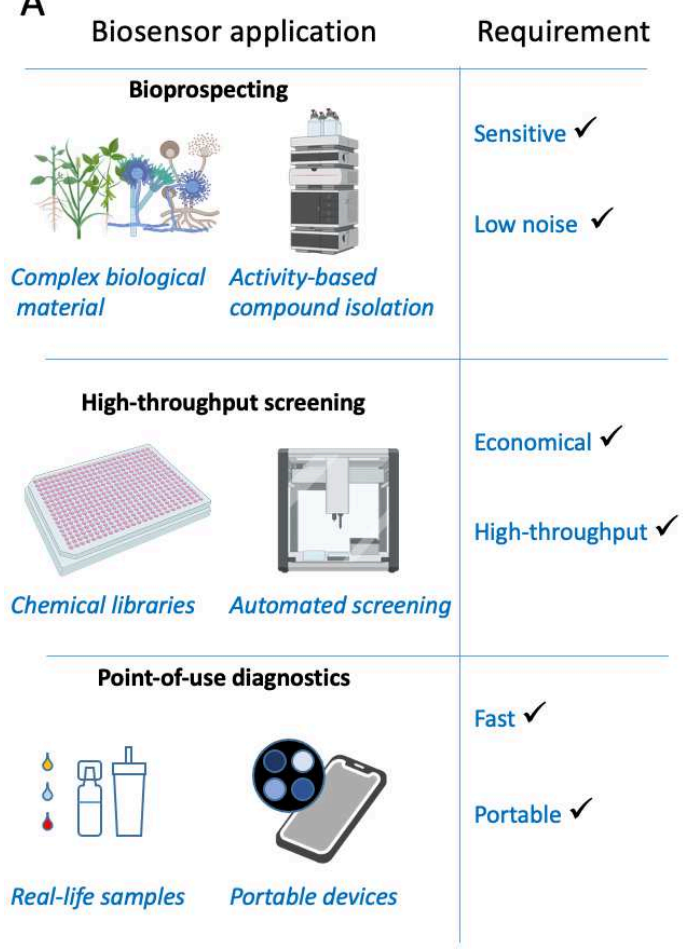

B

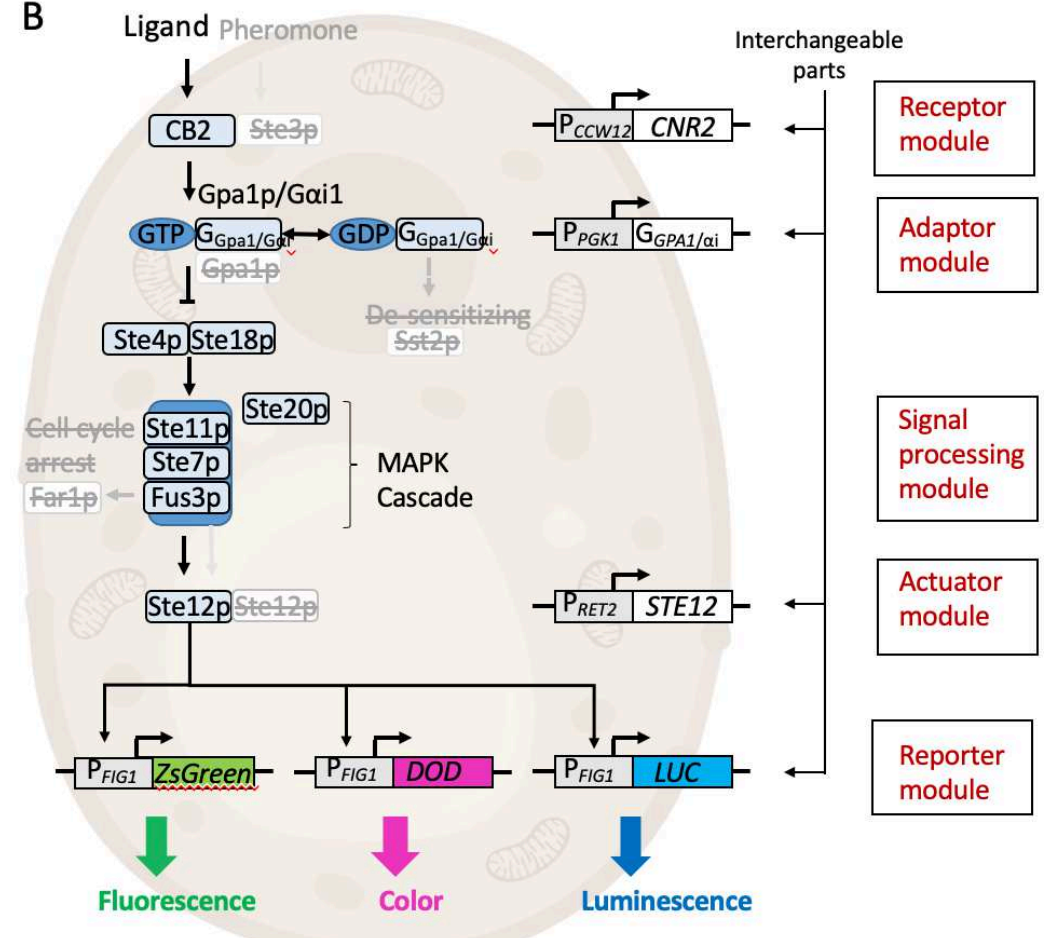

Figure 1. The CB2 cannabinoid biosensor design. (A) The biosensor was developed to enable diverse applications with specific requirements. For example, the bioprospecting of complex biological material requires the biosensor to be sensitive but with a low background. This is because the bioactive compounds are often present in minute amounts among high numbers of compounds potentially interfering with the detection. On the other hand, screening of chemical libraries requires a biosensor that is robust, economical, and amenable to highthroughput workflow. For this, fast growing and easy to prepare cells that can be handled with non-expensive material and equipment are desirable. In the case of a biosensor for point-of-use diagnostics outside the lab, this needs to be easy to use, fast, and operable by equipment available to non-experts. (B) The cannabinoid biosensor is based on a modular design. Interchangeable parts can be introduced into the receptor, adaptor, actuator, or reporter modules (red), while the native yeast G $\beta$ and Gy subunits and the MAPK cascade are used as a signalprocessing module without further modification. The parts are integrated into a chassis strain where genes encoding the yeast pheromone pathway components to be replaced (pheromone receptor STE3, Ga subunit GPA1, and pheromone pathway master regulator STE12) have been removed (strikethrough) alongside with SST2 (which returns $G \alpha$ to its inactive state) and FAR1 (which triggers cell-cycle arrest). This design enables functional insertion of different GPCR receptors by pairing them with the corresponding Gpa1p/Ga chimera. According to application requirements, the biosensor can be fitted with an optimal reporter construct including, for example a fluorescence, color, or luminescence reporter.

In this work, we develop a flexible modular cannabinoid biosensor by coupling the human CB2 receptor to the yeast pheromone-signaling pathway (Fig. 1B). We optimize the developed biosensor to achieve similar sensing dynamics than mammalian cell-based systems ${ }^{31}$ in a far more economical and userfriendly format. We further expand the performance of the system by developing dedicated color- and luminescence-based reporter strains to meet the specific requirements of different demanding 
97 applications and showcase the biosensor's performance in three case-studies. To demonstrate the 98 biosensor's high-throughput screening capacity, we present here the discovery of two novel CB2 99 agonists and four novel CB2 antagonists from a compound library of 1600 synthetic compounds. To 100 showcase the sensor's ability to cope with highly complex biological samples, we apply it in the 101 bioprospecting of 71 extracts derived from different parts of 54 different medicinal plants and describe 102 the bioactivity-guided isolation of a novel agonist of CB2, dugesialactone. Finally, we demonstrate the 103 use of a biosensor as a sensitive portable device for detecting cannabinoids from reconstructed saliva 104 samples.

105

106 Our results harness the extensive sensing repertoire of GPCRs to establish robust whole-cell 107 biosensors. This technology can now be extended to detect numerous other molecules, from small 108 compounds to proteins, enabling advanced biotechnological applications.

109 
Results

111 Constructing the chassis for the GPCR-based biosensor platform. The GPCR signaling

112 mechanism is inherently modular and can be abstracted in the form of five linearly connected modules

113 (Fig. 1B). The "input module" comprising the GPCR protein, the "adaptor module" that contains the 114 dedicated Ga protein, the "signal processing module" that encompasses the MAPK cascade, the 115 "actuator module" that contains the MAPK-controlled transcription factor, and, finally, the "output 116 module" that includes the activated genes. Our biosensor design takes advantage of this modular 117 structure to construct different biosensors by using a basic chassis (or platform) strain and integrating 118 different combinations of parts in the above-mentioned modules (Fig. 1B). This enables flexible setup 119 and functional optimization of the biosensor for different applications by shuffling different component 120 encoding genes and promoters in each module to find the best-performing configuration.

To enable this modular design, we first constructed the chassis strain by removing the genes encoding for pheromone pathway components that are to be replaced by custom parts (Table S1) (Fig S1). Thus, we knocked out the genes for the a-pheromone receptor (STE3; to be replaced by GPCR receptorencoding gene), the Ga subunit (GPA1; to be replaced by a Ga gene that is compatible with the chosen GPCR), and the pheromone response master regulator transcription factor (STE12; a prerequisite for removing GPA1). Additionally, we removed two genes that are detrimental to biosensor function (SST2 and FAR1). SST2 encodes a protein that contributes to returning $\mathrm{Ga}$ to its non-activated state $^{32}$, resulting in attenuated signaling through the pathway, and FAR1 causes yeast to enter cell-cycle arrest following pheromone sensing. This resulted in the chassis strain KM111, which serves as a basis for all subsequent biosensor strains.

Constructing the initial cannabinoid biosensor. The initial cannabinoid biosensor strain KM202 (Fig.

3) was constructed by integrating four genes (Fig S2, Forman et al., unpublished) into the chassis strain KM111 (Fig S3) (Table S1). These include the human cannabinoid receptor CB2 gene (CNR2), a hybrid gene encoding for a chimera between the yeast Ga protein and the five C-terminal amino acids of human 
Gai1 (Gpa1/Gai1) capable of linking the receptor to the downstream pathway ${ }^{2,3}$, the pheromone pathway master regulator (STE12), and the fluorescent reporter $\left(\right.$ ZsGREEN $\left.^{33}\right)$. To correctly balance the pathway components, the abovementioned genes were put under the control of specific promoters, according to the findings by Shaw and co-workers ${ }^{5}$. Thus, CB2 was put under control of the strong constitutive promoter $\mathrm{P}_{C C W 12}$, Gpa1/Gai1 under the medium/strong constitutive promoter $\mathrm{P}_{P G K 1}$, STE12 under the medium strength constitutive promoter $\mathrm{P}_{R E T 2}{ }^{34}$ and the reporter gene ZsGREEN under the native pheromone response promoter $P_{F I G 1}$ that is activated by Ste $12 p^{35}$.

To evaluate the performance of the initial biosensor, it was tested with a concentration series of the potent CB2 full agonist CP55940 resulting in a typically shaped dose response curve (Fig 2A). The calculated $\mathrm{EC}_{50}$ was $11 \mathrm{nM}$, which is in a comparable range as mammalian CB2 expression systems ${ }^{31}$. The limit of detection (LOD) was determined to be $100 \mathrm{pM}$ and the maximum signal to noise ratio (SNR) was 8.9:1. In order to test the usefulness of the biosensor in the detection of less potent agonists, we assayed the partial agonist (-)-trans- $\Delta^{9}$-tetrahydrocannabinol (THC). The corresponding dose-response curve (Fig. 2B) revealed values for $\mathrm{EC}_{50}$ of $1.8 \mu \mathrm{M}$, SNR of 3.1:1, and LOD of $100 \mathrm{nM}$. Thus, further optimization of the biosensor is required to enable the detection of low concentrations of less potent agonists.

Optimization of the basic biosensor. Biosensor sensitivity, evaluated here by the $\mathrm{EC}_{50}$ or LOD values, is mostly determined by the intrinsic properties of the receptor (affinity for the ligand) and the number of active receptors on the cell surface ${ }^{5,32}$. Previous work has demonstrated that the degree of membrane localization is an important bottleneck for heterologous GPCR functionality and differs greatly between heterologously expressed receptors. Appending the yeast mating factor a (prepro)secretion signal (MFaSS) to the $\mathrm{N}$-terminus of the receptor has been shown to enhance both the total expression and membrane localization of GPCRs in yeast ${ }^{36}$. Thus, in order to enhance the sensitivity and overall output of the cannabinoid biosensor, we constructed biosensor strain KM203 where CB2 is fused to MFaSS (Fig S4) and expressed from the strong yeast promoter $\mathrm{P}_{c c w 12 .}$ When the new biosensor was tested 
with CP55940 or THC and its dose-response curve was compared with that of strain KM202 (no

165

166

167

168

169

170

171

MFaSS), a clear improvement in biosensor sensitivity and output level was observed (Fig. 2A and B).

In the case of CP55940, the $\mathrm{EC}_{50}$ improved 3.7 times to $3 \mathrm{nM}$, the LOD decreased 5.6 times to $18 \mathrm{pM}$, and the maximum SNR improved to 12.2:1. Similarly, with $\mathrm{THC}$, the $\mathrm{EC}_{50}$ improved to $1.2 \mu \mathrm{M}$ (1.5-fold), LOD to $32 \mathrm{nM}$ (3.1-fold), and SNR to 5.3:1 (1.7 fold). Overall, the sensitivity and maximum output was improved to a level that this strain can be used for the detection of cannabinoids with a sensitivity on par with that of mammalian systems ${ }^{31}$.
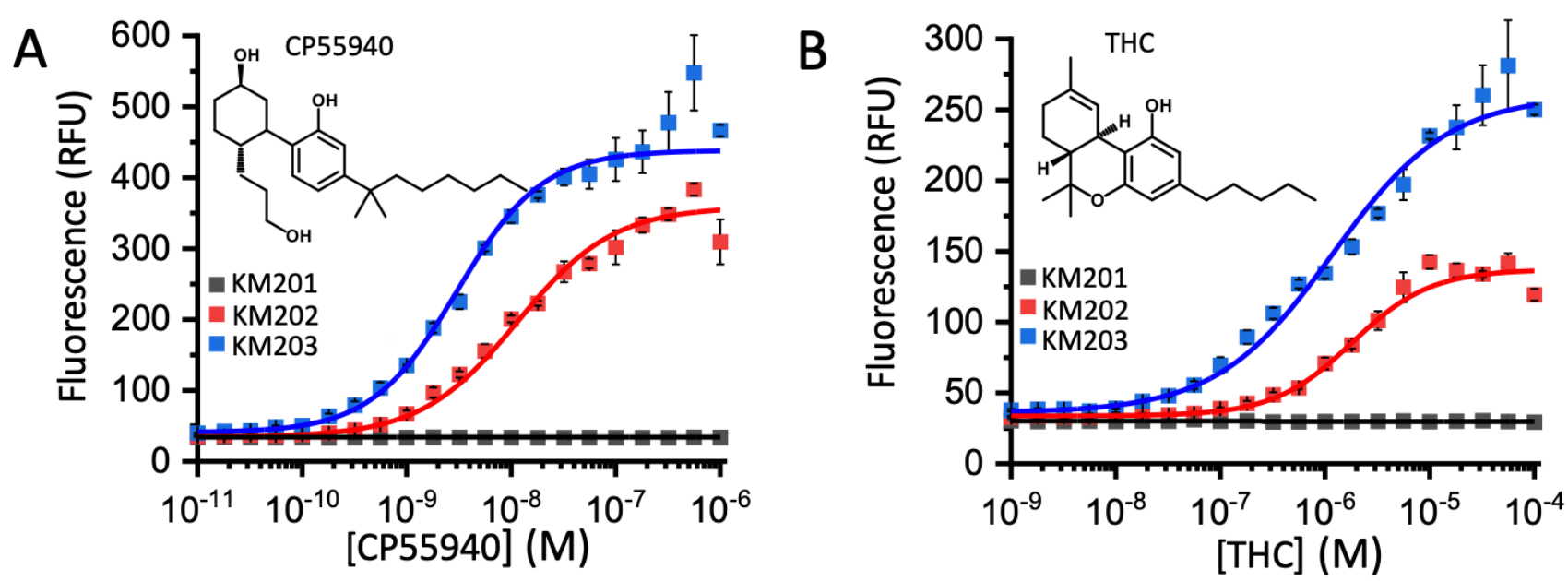

172

Figure 2. Detection of cannabinoids with the fluorescent output cannabinoid biosensor. (A) Incubation of the cannabinoid biosensor strain in the absence of CB2 receptor (KM201, black) with up to $1 \mu$ M CP55940 resulted in no increase of fluorescent output. Inducing biosensor strain KM202 expressing CB2 receptor (red) with 10 pM to $1 \mu \mathrm{M}$ CP55940 resulted in a typical sigmoidal dose response curve revealing an apparent $\mathrm{EC}_{50}$ of $11 \mathrm{nM}$ and $E_{\max }$ of $383 \mathrm{RFU}$. Furthermore, strain KM203 (blue) including CB2 appended with the mating factor a signal sequence showed a curve indicating higher sensitivity $\left(\mathrm{EC}_{50}\right.$ of $\left.3 \mathrm{nM}\right)$ and output (547 RFU). (B) Incubating the above-mentioned strains with THC resulted in similar dose response curves. Also here, KM201 (black) was not induced by the cannabinoid, whereas KM202 (CB2, red) showed an apparent EC $\mathrm{C}_{50}$ of $1.8 \mu \mathrm{M}$ and $\mathrm{E}_{\max }$ of 184 RFU. Respectively, KM203 (MFaSS, blue) higher sensitivity (EC50 $1.2 \mu \mathrm{M})$ and output ( $\mathrm{E}_{\max } 283 \mathrm{RFU}$ ).

Novel betalain-based colorimetric reporters for portable biosensors. To further improve the applicability of the developed biosensor, we focused on optimizing the performance of the output module. The current biosensor utilizes the fluorescent protein ZsGREEN, which provides a relatively direct relationship between reporter expression and signal magnitude (number of fluorescent molecules), but it is confined to measurements in a laboratory setting. To enable analysis in real-life settings, the performance of the output module can be enhanced in two ways. First, switching from 
fluorescence to an output that can be detected without a dedicated instrument, such as color or light, will enable portable applications. Second, using an enzyme-based reporter instead of fluorescence will add a signal amplification step, thus enabling detection with low-gain devices such as mobile phones.

Typical colorimetric reporters used in yeast include glycosidases ${ }^{37}$ and carotenoid biosynthesis genes ${ }^{24}$. we focused on betalains, the intense pigments found in red beet (Beta vulgaris) and constructed three novel reporter systems producing different colors.

A
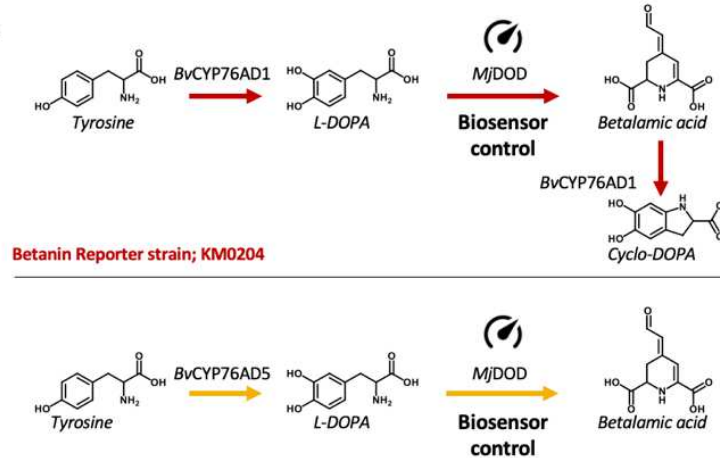

Betaxanthin Reporter strain; KM205

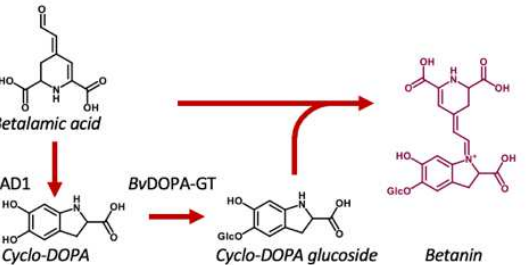

Cyclo-DOPA glucoside
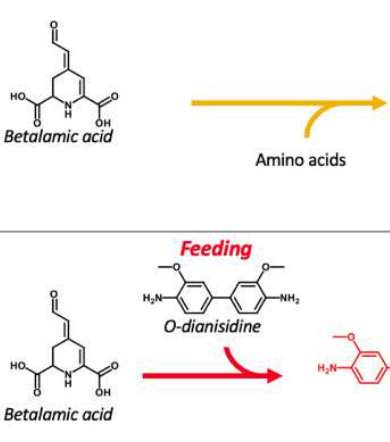

B

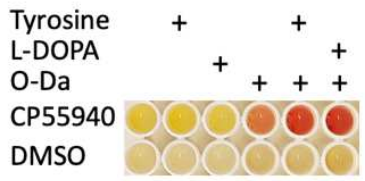

KM204

\section{D}

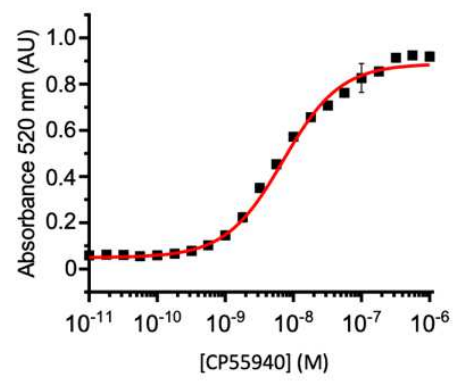

198

199

200

201

202

203

204

205

206

207

208

209

210

211

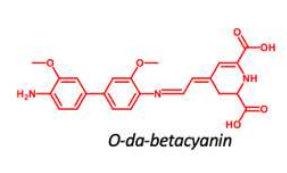

点

C

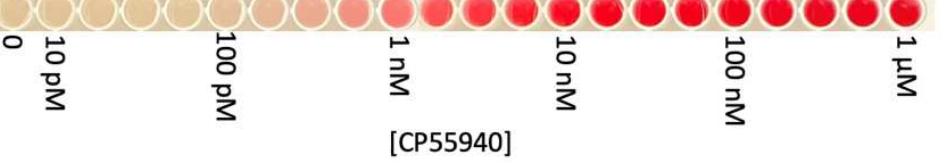

Figure 3. Novel betalain biosynthesis-based reporters. (A) The three betalain based transcriptional reporter systems were constructed based on three different biosynthetic pathways. In all the reporters, DOPA dioxygenase (MjDOD) was the reporter gene that is controlled by the biosensor and other genes were constitutively expressed. The betanin reporter is based on the strain KM204, where BVCYP76AD1 hydroxylates tyrosine into L-DOPA that is further oxidized to betalamic acid. It is then further oxidized by BVCYP6AD1 into cyclo-DOPA and glucosylated by DOPA-5-glucosyltransferase to cyclo-DOPA-glucoside. Finally, betalamic acid and cyclo-DOPA-glucoside can spontaneously condense to make betanin. The betaxanthin reporter, on the other hand, is based on strain KM205, where CYP76AD5 is used to hydroxylate tyrosine into L-DOPA. This is further oxidized into betalamic acid. In this case, this compound will spontaneously react with amino acids available in the cells to make betaxanthins. The O-dianisidine-betacyanin reporter is also based on strain KM205. In this case, however, L-DOPA is directly fed to the cells ensure a higher flux through the pathway when MjDOD is being expressed. Then, L-DOPA is oxidized by MjDOD to betalamic acid, which subsequently reacts strongly with O-dianisidine to produce O-da-betacyanin. (B) The addition of supplements to the strain KM205 has a dramatic effect on the color output. While the addition of 
O-da results in the appearance of a red color, supplementing with both O-da and L-DOPA results in the strongest 213 color. (C) The color produced by the O-da-betaxanthin reporter can be easily detected by eye. Inducing the strain $214 \mathrm{KM} 205$ with a dilution series ranging from $10 \mathrm{pM}$ to $1 \mu \mathrm{M} \mathrm{CP55940}$ for $16 \mathrm{~h}$ and supplementing it with L-DOPA and O-da results in different intensities of red, where the effect of 100 pM CP55940 could still be detected by eye. (D) The output of the O-da-betacyanin reporter can be quantified by measuring the absorbance of the pigment at $520 \mathrm{~nm}$. Measuring the effect of the above-mentioned CP55940 range resulted in a typical dose-response curve with an apparent $\mathrm{EC}_{50}$ of $1.8 \mathrm{nM}$.

The group of betalains includes two categories of compounds, the purple-red betacyanins and the orange betaxanthins (Fig S5). To produce the betacyanin betanin in yeast, we introduced the tyrosine hydroxylase/cyclase from $B$. vulgaris (BvCYP76AD1), the DOPA dioxygenase from Mirabilis jalapa (MjDOD), and the DOPA 5-glucosyltransferase from B. vulgaris (BvDOPA5GT) ${ }^{38}$ to construct biosensor strain KM204 (Fig 3A, Table S1). In this strain, MjDOD serves as the effective reporter gene controlled by the pheromone responsive $\mathrm{P}_{F I G 1}$ promoter, whereas BVCYP76AD1 and BVDOPA5GT were

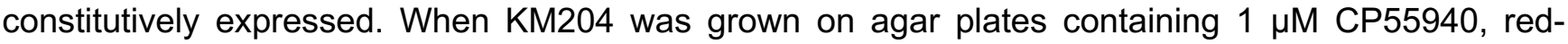
colored colonies were clearly visible suggesting that the biosensor was functional (Fig S6). However, color build-up in liquid cultures of KM204 was slow (>16h), suggesting that although using betanin as reporter could be useful in library screening experiments, a faster responding reported would be preferable for a portable biosensor. Thus, we turned to the yellow betaxanthins and constructed a biosensor strain (KM205) co-expressing MjDOD controlled by the $\mathrm{P}_{F / G 1}$ promoter together with the tyrosine hydroxylase BvCYP76AD5 from B. vulgaris (Fig 3A), as this combination has been shown to produce betaxanthins in yeast ${ }^{38}$. This time, in addition to obtaining yellow-colored colonies on CP55940containing agar plates (Fig S6), measuring the absorbance of the KM205 culture supernatant showed a dose-dependent biosensor signal (Fig. S7).

Encouraged by this finding, we continued to develop a biosensor with output distinguishable by eye. The betalain precursor betalamic acid can readily react with several primary and secondary amines to produce compounds with intense colors ${ }^{38}$. Thus, to develop a reporter with strong red color, we supplemented cultures of KM205 with $0.5 \mathrm{mM} \mathrm{O-dianisidine} \mathrm{(O-da)} \mathrm{to} \mathrm{obtain} \mathrm{O-da-betacyanin.}$ 241 Furthermore, because it has been shown that betalain production can be improved by adding tyrosine 
in the yeast media, we also added either tyrosine or L-DOPA (the next step in the pathway) to boost color production. A clearly enhanced color signal was obtained (Fig 3B). As KM205 with O-da and LDOPA showed the most distinguishable color, we chose to use this combination in the rest of this work and refer to it as "the betalain reporter". Testing the betalain reporter strain with a dilution series of CP55940 revealed that the color signal was detectable by eye down to 100 pM (Fig 3C). Quantification of the color signal from this series by measuring absorbance at O-da-betacyanin peak absorbance wavelength, $520 \mathrm{~nm}$ (Fig S6) showed an apparent EC50 of $7 \mathrm{nM}$ (Fig 3D), indicating similar sensitivity with the fluorimetric strain KM203.

A luminescence reporter improves dynamic range and response time. Although our developed

fluorescence and betalain reporter strains are already well suited for cannabinoid detection inside and outside the lab, some key applications such as determination of cannabinoids in complex biological material present additional challenges, as many biological samples are likely to contain colored or fluorescent molecules. Similarly, yeast cells, under specific conditions may display high levels of autofluorescence or produce small amounts of colored molecules. Thus, developing a biosensor with a reporter less prone to background noise would be beneficial. In addition, shortening the response time for the biosensor will be desirable for certain applications. We set out to developing a new biosensor strain to address both requirements.

We opted for a luminescence-based reporter because its output is light, which avoids background or interference from the sample or the chassis. To achieve a strong luminescence output, we chose the NanoLuc luciferase, as it has been shown to produce the brightest signal among similar enzymes $^{39}$. We constructed the luminescence reporter strain KM206 (Table S1) by placing NanoLuc expression under the control of $\mathrm{P}_{F I G 1}$ in the reporter module. When tested with CP55940, the response time was considerably faster, producing sufficient output after $1 \mathrm{~h}$ of induction to be measured with a cellular phone camera (Fig. 4A). Moreover, the background signal was minimal $(0.1 \%$ of the maximum signal) (Fig. 4B). Moreover, we found that the biosensor was functional at DMSO concentrations up to 
facilitate its use in different applications with different technical requirements. To assess the biosensor's performance with less potent cannabinoids that might also be more relevant for real-life applications, we also produced dose response curves for $\mathrm{THC}$, its liver metabolite 11-OH-THC, and the synthetic cannabinoid JWH-018 or "Spice" (Fig. 4C-E). "Spice" or "K2" are Illegal synthetic cannabinoid preparations that have been increasingly found on the street market. These compounds cannot be from patients or users.

A

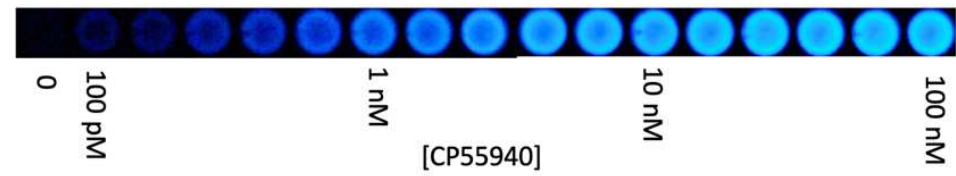

B

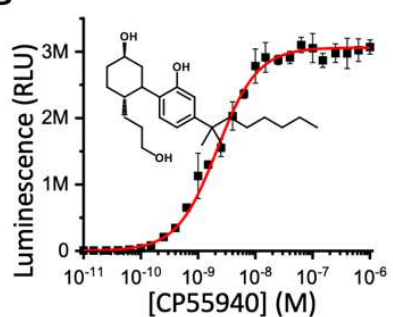

278
C

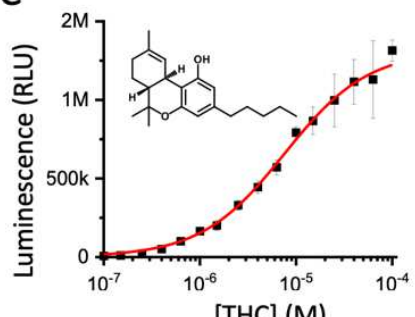

$[\mathrm{THC}](\mathrm{M})$
D

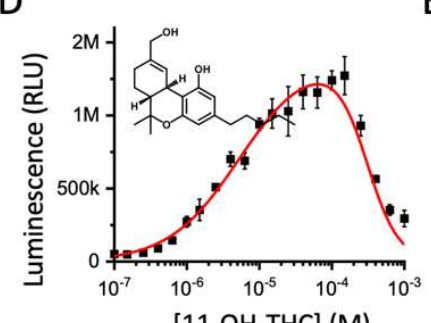

[11-OH-THC] (M)

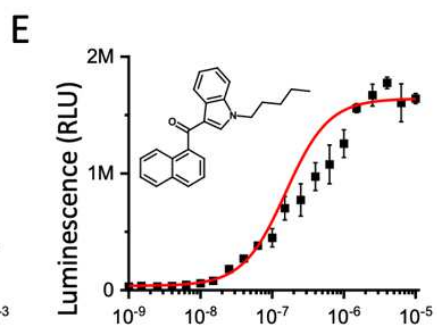

[JWH-018] (M)

Figure 4. A cannabinoid biosensor with a luciferase reporter. (A) The biosensor strain KM206 employs a luciferase reporter. It was incubated with a dilution series of CP55940 ranging from $100 \mathrm{pM}$ to $100 \mathrm{nM}$ and photographed it with a cell phone camera. This resulted in a clearly visible signal until 100 pM CP55940. (B) The response of the strain KM206 to different cannabinoids was determined by incubating with a dilution series of the strong synthetic cannabinoid CP55940. (C) Dose response curve with THC, the main cannabinoid from cannabis. (D) Dose response curve with 11-OH-THC, the liver metabolite of THC. (E) Dose response curve of the structurally unrelated strong synthetic cannabinoid JWH-018. The resulting luminescent signal was measured using a plate reader.

The two new output modules improve biosensor performance. Having set up two new biosensor strains with different reporters and performance characteristics, we sought to establish which biosensor was better suited for each of the different possible applications. For example, portable applications for use in the field require short response time and a reporter that can be measured without professional equipment. A biosensor for drug screening applications should be compatible with a high-throughput 
format and preferably economical. On the other hand, identifying cannabinoids in complex biological

material requires low background and high sensitivity to detect very low concentrations of compounds.

A

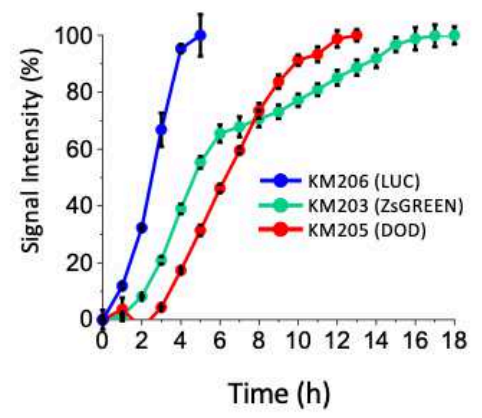

B

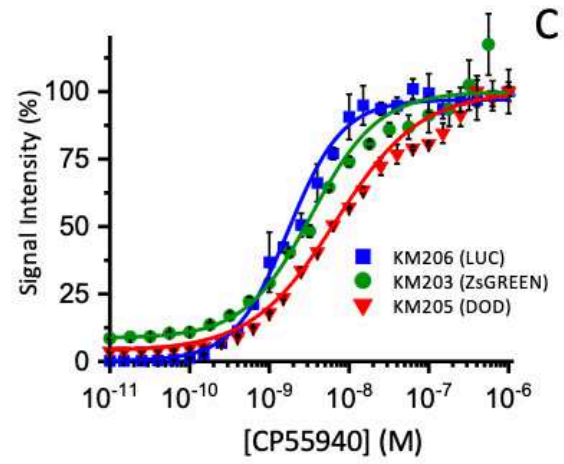

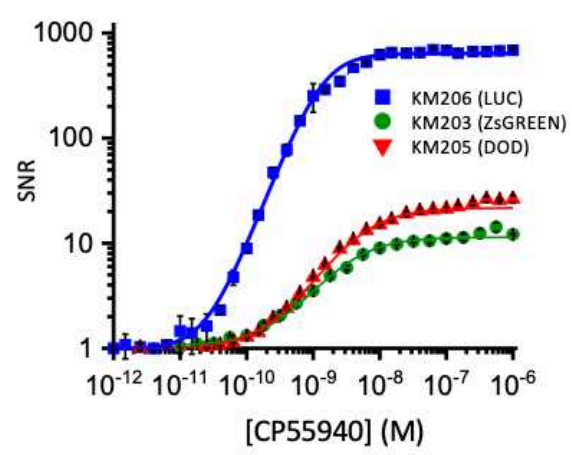

297

298

299

300

301

302

303

304

305

306

307

308

309

310

311

312

313

314

315

Figure 5. Comparison of the characteristics of three biosensor strains with different reporters. (A) In order to compare the response time of the biosensor strains KM203/fluorescence, KM205/betalain color (with supplements) and KM206/luminescence, their outputs were measured once per hour after induction with $1 \mu \mathrm{M}$ CP55940 until they reached saturation. $\mathrm{T}_{50}$ (time to reach $50 \%$ of the maximum signal) was determined to be 2 h, $3.5 \mathrm{~h}$ and $1 \mathrm{~h}$ hours for KM203, KM205 and KM206 respectively. (B) Sensitivity of each sensor was determined from $\mathrm{EC}_{50}$ and LOD values. This information was calculated from dose-response curves resulting from incubation of each biosensor with a dilution series of CP55940 from 10pM to $1 \mu \mathrm{M}$. As expected, these values were in a similar range for all three strains: $\mathrm{EC}_{50}$ values were $3.37 \mathrm{nM}, 6.79 \mathrm{nM}$, and 1.78; LOD values $18 \mathrm{pM}, 63 \mathrm{pM}$, and 40 pM; for KM2013, KM205 and KM204, respectively. (C) Comparison of the maximum signal to noise ratios calculated from the above-mentioned dose-response curve revealed that KM206 had a far superior dynamic range (up to 1000:1) compared to that of KM203 (14:1) and KM205 (26:1).

We started by measuring the response time of each of the strains with a saturating amount $(1 \mu \mathrm{M})$ of CP55940 (Fig 5A). $\mathrm{T}_{50}$, the time required to reach 50\% of maximum activity for the luminescence strain was $2.5 \mathrm{~h}$, which was clearly shorter than for the fluorescence or betanin reporter strains $(4.5 \mathrm{~h}$ and 6.5 h respectively). Comparison of the CP55940 dose response curves revealed similar sensitivity for all three biosensors, as this is inherent to the CB2 receptor (Fig 5B).

\begin{tabular}{|c|c|c|c|c|c|c|c|c|c|c|c|c|c|c|}
\hline \multirow[t]{2}{*}{ Strain } & \multirow[t]{2}{*}{ Type } & \multirow{2}{*}{$\begin{array}{l}\text { Speed } \\
\text { Time } \\
50 \%(h)\end{array}$} & \multirow{2}{*}{$\begin{array}{l}\text { Dyn range } \\
\text { Max SRN }\end{array}$} & \multicolumn{2}{|c|}{ Sensitivity } & \multicolumn{3}{|c|}{ Operational range } & \multicolumn{2}{|l|}{ Screening } & \multicolumn{2}{|c|}{ Bioprospecting } & \multicolumn{2}{|c|}{ Portable applications } \\
\hline & & & & $\begin{array}{l}\text { LOD } \\
\text { (pM) }\end{array}$ & $\begin{array}{r}\text { EC50 } \\
(n M)\end{array}$ & $\begin{array}{l}\mathrm{EC} 20 \\
(\mathrm{nM})\end{array}$ & $\begin{array}{r}\text { EC80 } \\
(n M)\end{array}$ & (fold) & Economical & $\begin{array}{l}\text { HTS } \\
\text { format }\end{array}$ & $\begin{array}{l}\text { Low } \\
\text { background }\end{array}$ & Sensitive & Portable & Fast \\
\hline KM203 & Fluorescence & 3.5 & 26 & 18 & 3.37 & 0.88 & 13 & 14.8 & +++ & +++ & + & +++ & + & ++ \\
\hline KM205 & Color & 6.5 & 14 & 63 & 6.79 & 1.25 & 36.7 & 29.4 & +++ & + & + & +++ & +++ & + \\
\hline KM206 & Luminescence & 2.5 & 980 & 40 & 1.78 & 0.91 & 5.3 & 5.8 & ++ & +++ & +++ & +++ & +++ & +++ \\
\hline
\end{tabular}

Table 1. Comparison of the biosensor strains. Characteristic properties of the three biosensor strains were calculated from time series and dose-response curves with CP55940. This revealed that the biosensor strains have different sensitivities, response times and dynamic ranges. In addition, the fundamental differences of the 
reporters (fluorescence, color and luminescence) contribute to differences in ease of signal quantification and necessary equipment. According to the previously discussed requirements for different applications, strains KM206 and KM203 were deemed best suited for HTS screening, while KM206 is the best choice for bioprospecting. For portable applications both KM204 and KM206 are favorable.

When we compared the dynamic range (maximum SNR), it was evident that the luminescence strain

(KM206) has a clearly superior dynamic range, up to 1000:1, whereas betalain (KM205 and

fluorescence (KM203) strains only reached 26:1 and 14:1, respectively (Fig 5C; Table 1). These results

suggest that the luminescence strain is far superior for quantitative analysis, such as dose response

measurements. It is also the reporter of choice for applications that require fast response. The betalain

strain on the other hand has a broad operational range and it is portable and economical without compromising in sensitivity. The suitability of these biosensors for different applications is further exemplified in the three case studies that follow.

\section{Case study: Discovery of novel CB2 agonists and antagonists by high-throughput screening of}

compound libraries. Currently, most new GPCR-targeting drugs are discovered by high-throughput for non-specialist, limited-budget labs, we based our method on the inexpensive, open source, liquid handling robot Opentrons OT-2 and a common plate reader (Molecular devices M5) in 384-well plate format (Fig. 6A).

As proof-of-concept, we screened 1600 randomly chosen compounds from the total $>55000$ compounds present in the chemical compound collection of the "Chemical biology and HTS" facility of the University of Copenhagen (https://cbhts.ku.dk/chemical-compound-collection/). For simplicity of analysis and low cost, we started developing the high-throughput screening method by performing an agonist screen 
using the betalain biosensor strain (KM205) as it directly displays color signal and does not require cell lysis. Following automated sample preparation and incubation for $16 \mathrm{~h}$ at $30^{\circ} \mathrm{C}$, visual inspection of the plates revealed several wells with distinguishable color build-up (Fig S9). Among them, two wells clearly stood out, indicating the presence of CB2 agonists (named AGO1 and AGO2 here, Table S2).

352

We repeated the library screening with the luminescence biosensor strain (KM206) to compare the two methods. The luminescence-based screen required incubation of only $3 \mathrm{~h}$, but also required addition of the cell lysis reagent and luciferin and detection of luminescence in a plate reader. The results confirmed the hits obtained using the colorimetric strain. As shown in Fig 6B, AGO1 and AGO2 triggered very strong activation (10- and 80-fold signal over background, respectively). We used the luminescence biosensor to obtain dose response curves of these compounds and determined $\mathrm{EC}_{50}$ values of $200 \mathrm{nM}$ and $2 \mu \mathrm{M}$, respectively (Fig 6C and 6D).

Screening for receptor antagonists entails competitive inhibition assays where the presence of 362 antagonists results in reduced biosensor activation against a background of activated biosensors. Here, accurate quantification of biosensor activity is important. Thus, we chose to use the luciferase biosensor strain. Several potential antagonists were identified by looking for compounds that together with CP55940 resulted in a lower biosensor signal compared to the agonist CP55940 alone (Fig 6E). From these, we selected seven potential antagonists (ANT1-ANT7) for further experiments. To verify that the effect of the putative antagonists is specific to the CB2 receptor and not a non-specific effect, we built a control strain, KM207, by replacing CB2 with the A2A adenosine receptor (Fig S10) and tested it with each of the candidates. The control strain was activated by adenosine as expected, so we conclude there were no non-specific effects on the biosensor (Fig S11). Finally, dose response curves of the most potent antagonists (ANT2 and ANT5 Table S2) were obtained and their apparent IC $_{50}$ values were determined to be $29 \mu \mathrm{M}$ and $710 \mathrm{nM}$, respectively (Fig 6F and G). All identified agonists and antagonists are new compounds that have not been previously identified to modulate CB2. 

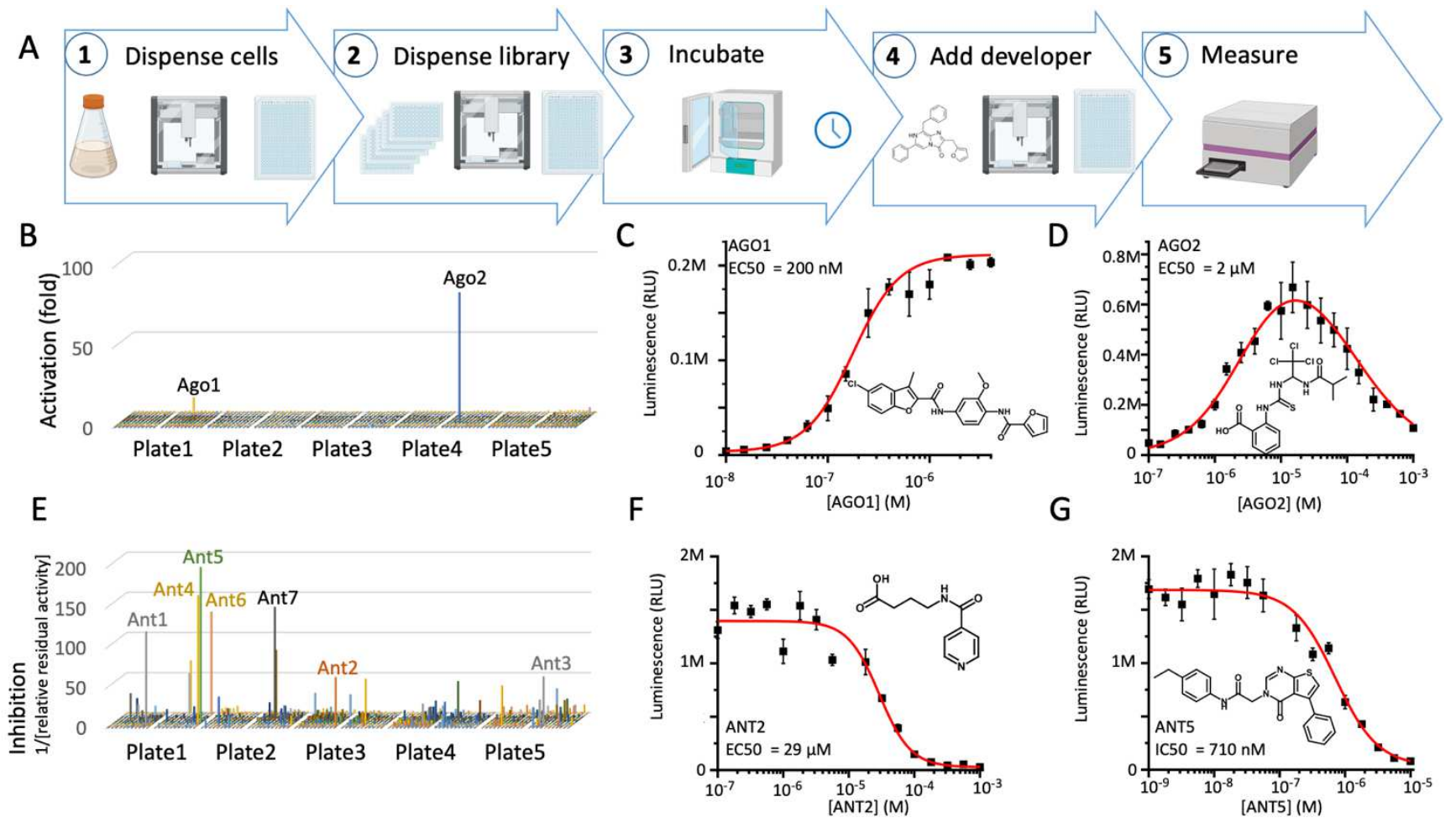

Case study: Discovery of novel phytocannabinoids by activity-guided bioprospecting. To further showcase the capabilities of the developed biosensor, we tested it in a problem with higher difficulty, screening for active compounds in complex biological samples (Fig 7A). Compared to pure compounds (as in the case of compound libraries), the use of biological material usually poses several additional challenges, such as low levels of analytes, and presence of compounds interfering with different components of the biosensor and the analysis. For this challenge, we chose the biosensor using

Figure 6. High-throughput screening applications. (A) The high resolution and low background of the KM206 cannabinoid biosensor strain makes it well suited for high-throughput screening of for GPCR ligands. The HTS screening workflow consists of (1) robotically dispensing the biosensor strain into 384 well plates, followed by (2) dispensing the library into these plates, (3) incubating for $3 \mathrm{~h}$, (4) adding the developer solution (lysis buffer with luciferin) to the cells and (5) measuring luciferase activity with a plate reader. (B) An agonist screen of the library led to the discovery of 2 novel CB2 agonists with markedly higher luciferase signal than the background. (C) $A$ dose-response curve for $A G O 1$ revealed an $E_{50}$ of 200 nM. (D) In the case of AGO2, a dose-response curve showed an $\mathrm{EC}_{50} 2$ of $\mu \mathrm{M}$. These results clearly validate the hits from the screening experiments. (E) An antagonist screen of the library was performed by adding the library on top of cell supplemented with $2 \mathrm{nM}$ CP55940. Here, compounds that resulted in lower luciferase activity than that of CP55940 alone were defined as hits (inhibitors). Their potency was calculated as [relative residual activity] ${ }^{-1}$. This revealed several potential antagonists (ANT1-7). (F) A dose-response curve for ANT2 revealed an $\mathrm{IC}_{50}$ of $29 \mu \mathrm{M}$. (G) For ANT5, a dose-response curve showed an $\mathrm{IC}_{50}$ of $710 \mathrm{nM}$ ). luminescence as reporter, because colored and fluorescent molecules typically present in plant extracts may mask fluorescent or colorimetric reporter output. 


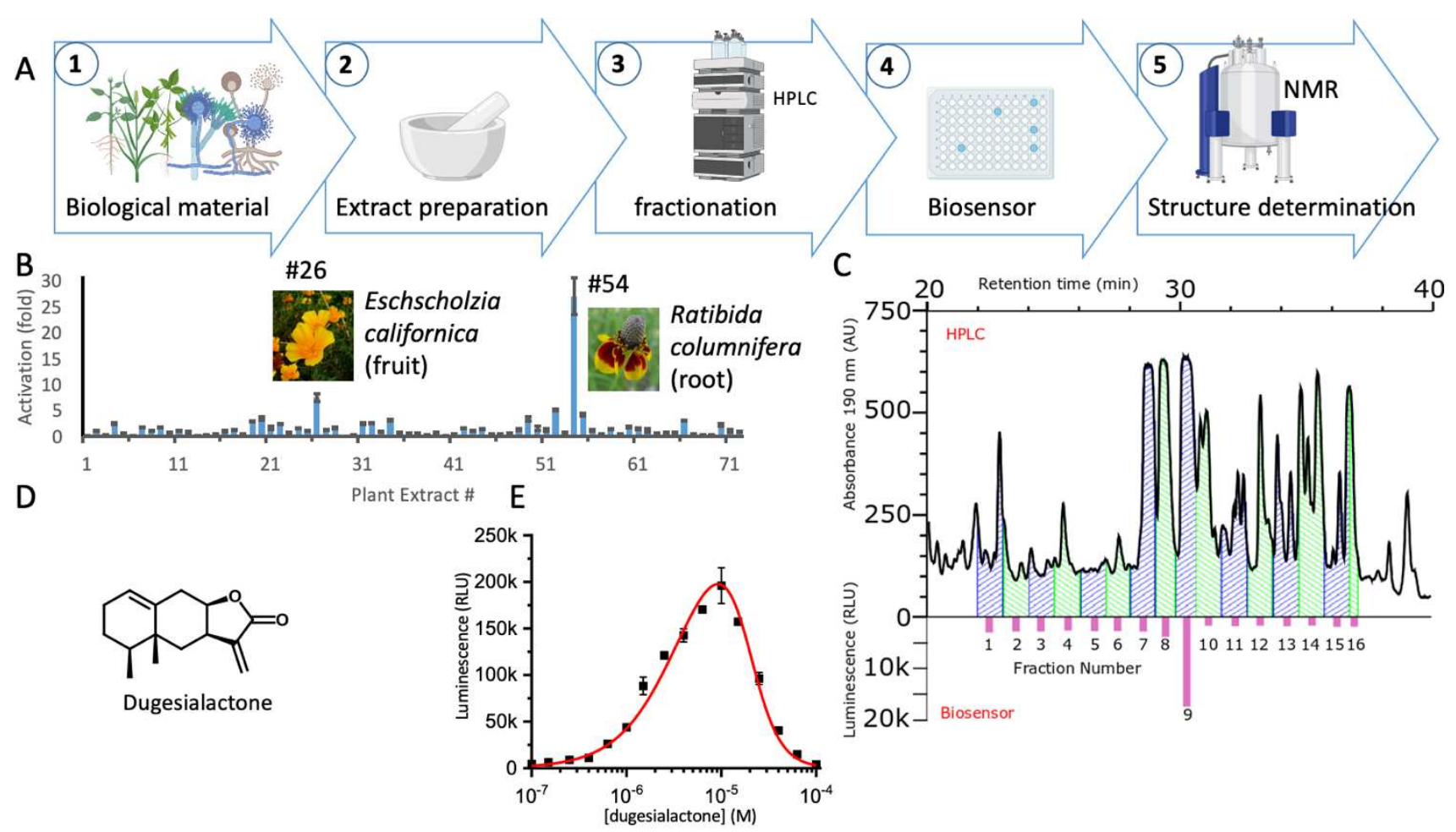

Figure 7. Bioactivity based discovery of GPCR ligands from complex biological samples. The high sensitivity of the biosensor enables the discovery of GPCR ligands from complex mixtures. (A) The bioprospecting workflow consists of (1) selection of biological material, (2) preparation of extract, (3) fractionation of extract using the chromatographic method of interest, (4) assaying the fractions using the biosensor strain and (5) structure determination of compounds in the fraction. Depending on the degree of separation of the compounds in the initial fractionation, one or more additional fractionation and biosensor assay steps can be performed to ensure purity of the target compound. (B) Assaying 71 plant extracts with the biosensor strain KM206 revealed two plant extracts that clearly activate CB2. (C) In order to purify main cannabinoid compound from the $R$. columnifera extract, this was fractionated into 16 fractions by preparative HPLC and each fraction was assayed with the biosensor to find the cannabinoid containing fractions. (D) The structure of the purified compound, dugesialactone, was determined by NMR. (E) The biosensor strain KM206 was incubated with a $10 \mathrm{nM}$ to $100 \mu \mathrm{M}$ dilution series of dugesialactone. The resulting dose response curve indicates an EC50 of $1.5 \mu \mathrm{M}$.

We started by screening 71 methanol extracts from different parts of 51 randomly chosen Mexican traditional medicinal plants (Table S3). The analysis revealed two plant extracts, namely those from Ratibida columnifera (root) and Eschscholzia californica (fruit), standing clearly above the background, showing a 27 -fold and 7 -fold increase in signal respectively (Fig 7B). To isolate the most active cannabinoid compounds from the $R$. columnifera extract, we used again the biosensor to perform bioactivity-guided fractionation. First, the extract was divided into 16 fractions by preparative High Performance Liquid Chromatography (HPLC) and dilutions of each fraction were assayed with the luciferase biosensor strain (Table S5, Fig 7C). Most of the active cannabinoids were present in fraction 9. Therefore, this fraction was further separated by prep HPLC (Fig S12) and, finally, the purified compound was submitted to (nuclear magnetic resonance) NMR analysis (Table S6). This revealed that the active compound is the sequiterpene lactone, dugesialactone (DL) (Fig 7D). DL was previously 
isolated from another Mexican plant, Dugesia mexicana ${ }^{40,41}$, but its function as phytocannabinoid was never identified. A dose response curve of DL revealed an apparent $\mathrm{EC}_{50}$ of $2 \mu \mathrm{M}$ (Fig 7E). Interestingly, at concentrations above $10 \mu \mathrm{M}, \mathrm{DL}$ acts as an inverse agonist. We confirmed that the effect of DL was specific to the CB2 receptor by testing DL with the A2A biosensor strain, as described in the previous section for the library hits (Fig S14).

429

Case-study: Portable biosensors for point-of-use applications. In order to expand this technology into consumer and non-specialist applications, a portable biosensor that does not require the use of lab equipment or expertise is needed. In this case, the biosensor must be configured so that it can be read by eye or with common devices such as a cell phone. Here, we develop two portable biosensor devices, one based on the betalain reporter strain, and another based on the luminescence reporter strain, and compare their performance using real-life samples.

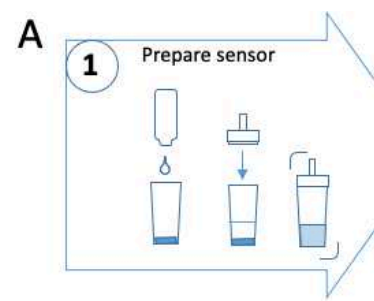

B
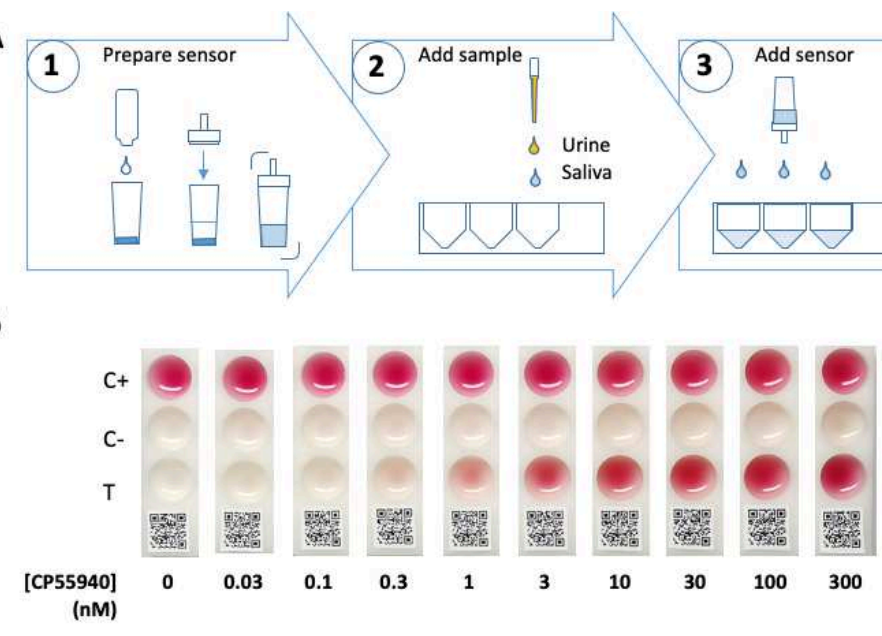
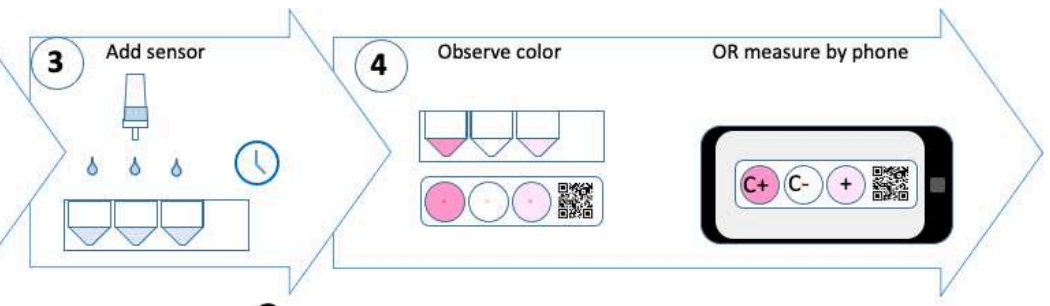

C

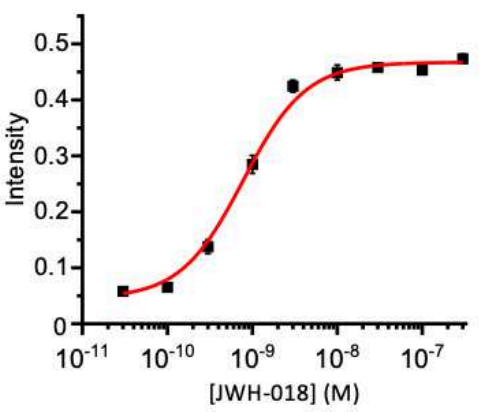

Figure 8 The portable colorimetric biosensor. (A) The cannabinoid biosensor was configured into a portable biosensor the output of which can be monitored by eye. This biosensor workflow using the colorimetric strain KM205 consists of (1) Preparing the biosensor strain by activating it with concentrated media (this solution also includes the DOPA and O-da supplement), (2) adding the sample to be measured in the portable biosensor device, (3) adding the activated biosensor yeast to the device and incubating 4-16 $\mathrm{h}$, (4) observing the color that was produced or measuring the signal by cell phone camera. (B) To test the portable biosensor workflow, a concertation series of CP55940 from $30 \mathrm{pM}$ to $300 \mathrm{nM}$ was added to the test wells ( $\mathrm{T}$ ) in of 10 biosensor devices. Positive control wells (C+) in each device had 300 nM CP55940 each and no negative control wells (C-) no cannabinoid. After addition of the biosensor and $16 \mathrm{~h}$ incubation the color in test wells could be detected by eye down to 30 pM CP55940. (C) To quantify the biosensor signal for the portable colorimetric biosensor the above experiment was made in triplicate and the O-da-betacyanin pigment measured from a cellular phone photograph (see methods). This resulted in a typical dose-response curve. 
452 We developed a dedicated bar-coded device for easy sample handling and an easy to implement 453 workflow that does not require scientific training (Fig 8A). According to this workflow, first, the sample 454 is applied to the sample well, and then the biosensor is mixed with the activating reagent (concentrated 455 minimal media) and applied to the dedicated device (test sample; T) (Fig 8A). The control wells contain 456 either a known amount of a dried cannabinoid (positive control; $\mathrm{C}+$ ) or no cannabinoid (negative control; 457 C-). After incubation for 16h, the activated biosensor can be readily read by eye (Fig 8B). 458 459 Although this method is mostly intended for binary cannabinoid detection, we also developed method 460 for quantifying cannabinoids using a cell phone camera and RGB color analysis. The biosensor output 461 is measured from a photograph as the average "redness" $(R)$ of the sample (see methods). A dose462 response curve for CP55940 measured by cell phone (Fig 8C) shows close resemblance to one 463 measured by plate reader.

464

465 Overall, we developed an efficient, economic, and robust biosensor that involves a simple workflow that 466 can be carried out by a non-expert. This biosensor characteristics enable the facile parallel processing 467 of large numbers of samples required for mass testing, e.g., quality control of cannabis products or 468 screening samples at customs or sports events. 

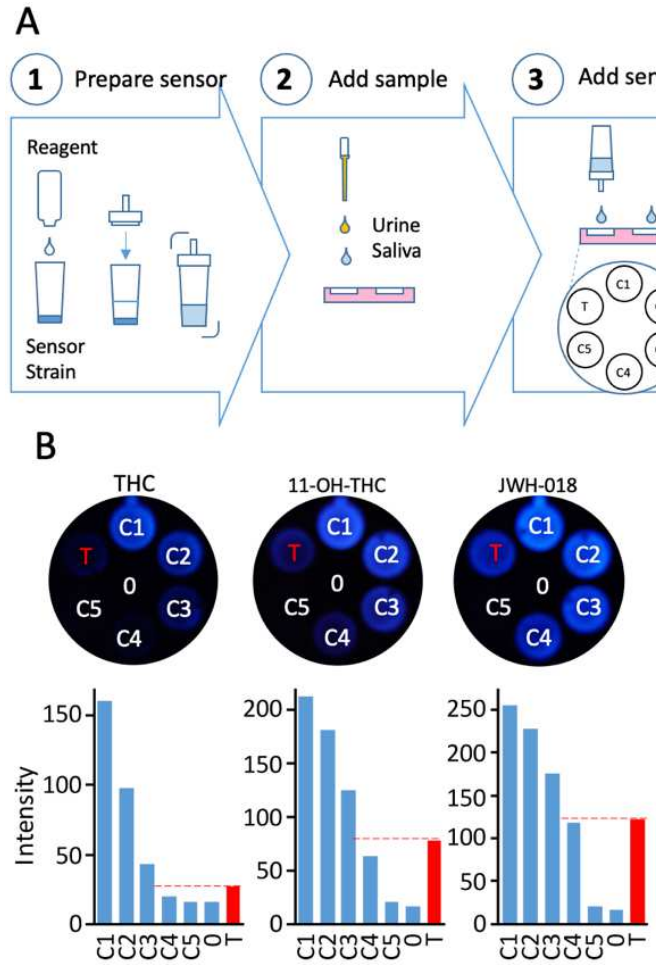

(3) Add sensor

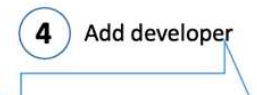

(5) Assemble device

(6)

Clip on phone and measure
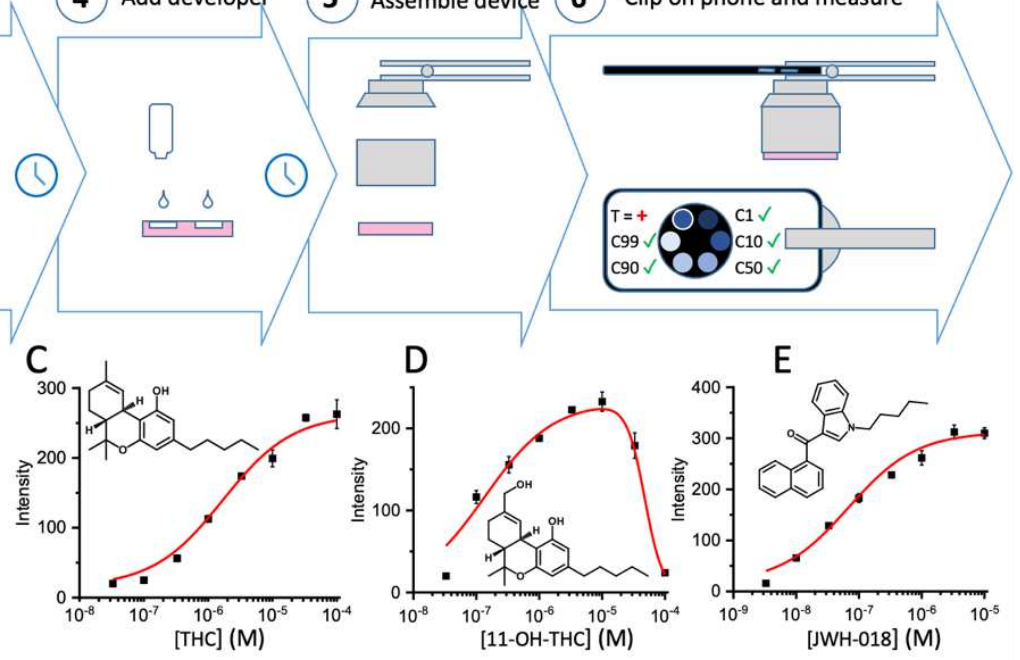

E

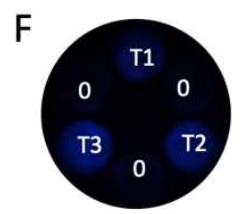

Figure 9. The portable luminometric biosensor. (A) The cannabinoid biosensor was configured into a portable biosensor that can be read by common equipment such as a cell phone. This biosensor using the strain KM206 workflow consist of (1) Preparing the biosensor strain by activating it with concentrated media (2) adding the sample to be measured in the portable biosensor device, (3) adding the activated biosensor yeast to the device and incubating $3 \mathrm{~h}$, (4) adding the developer solution (lysis buffer and luciferin), (5) assembling the rest of the portable biosensor device with a cellular phone and (6) measuring biosensor output with the phone. To test the workflow with the device dose response curves were produced by incubating the strain for $3 \mathrm{~h}$ in the device in the presence of (B) $33 \mathrm{pM}-100 \mu \mathrm{M}$ of the canonical cannabinoid THC, (C) $33 \mathrm{pM}-100 \mu \mathrm{M}$ of its liver metabolite 11$\mathrm{OH}-\mathrm{THC}$, or (D) $3.3 \mathrm{pM}-10 \mu \mathrm{M}$ of the synthetic narcotic JWH-018 or "spice". (E) Cannabinoid concentrations can be evaluated by measuring light intensity in the test well $(T)$ and comparing with calibration points (C1-5) of the respective compounds. Here, $150 \mathrm{nM}$ THC, $150 \mathrm{nM}$ 11-OH-THC or $15 \mathrm{nM} \mathrm{JWH-018} \mathrm{(red} \mathrm{bars)} \mathrm{show} \mathrm{signal}$ intensities placing them between calibration points for $100 \mathrm{nM}$ and $330 \mathrm{nM}$ THC, $100 \mathrm{nM}$ and $330 \mathrm{nM}$ 11-OH-THC or 10-33 nM JWH-018 respectively. (F) In order to test the portable biosensor with more real-life-like sample, reconstituted saliva spiked with $100 \mathrm{nM}(\mathrm{T} 1), 330 \mathrm{nM}(\mathrm{T} 2)$ and $1 \mu \mathrm{M}$ (T3) THC was assayed ( $0=$ no cannabinoid). (G) Measuring signal intensity from these cannabinoids contain wells shows a clear difference to no cannabinoid wells.

Whereas the colorimetric biosensor strain is well suited for the qualitative detection of cannabinoids from samples, some portable applications such as regular monitoring of cannabinoids in samples from patients treated with medicinal cannabis require the sensitive, quantitative measurement of compounds and a shorter development time. Thus, we developed a portable biosensor workflow based on the luminescence reporter strain and a purpose-built biosensor device (Fig 9A). This workflow consists of 
494 all wells. The biosensor device is assembled by attaching an adaptor ring and a clip-on cell phone macro 495 lens (here, Xenvo Clarus 15x) creating a closed, standardized, environment for image acquisition. 496 Finally, the sample image is acquired and analyzed by software that quantifies biosensor output by 497 measuring intensity of the blue channel of the resulting raw picture. By measuring the signals from the 498 five calibration wells, a reference curve can be created and used to normalize the measurements 499 between biosensor batches. Comparing sample signal to that of the calibration points can be used to 500 evaluate the concertation of a cannabinoid (Fig 9B). Using this workflow, we generated dose response 501 curves for THC (Fig 9C), its liver metabolism product 11-OH-THC (Fig 9D), and the synthetic 502 cannabinoid JWH-018 (Fig 9E). The portable biosensor-derived curves showed similar responses than 503 when measured in a laboratory setting with a luminometer (plate reader).

504

505 Finally, we demonstrate the usefulness of the portable biosensor using simulated human samples. 506 Following cannabis consumption, THC and/or its metabolic products 11-OH-THC, 11-oxo-THC and 11$507 \mathrm{COOH}-\mathrm{THC}$ can be found in urine and saliva in nano to micromolar concentrations ${ }^{42}$. To simulate 508 cannabinoid-containing human samples, artificial saliva was supplemented with THC according to 509 concentrations found in authentic samples and assayed according to the portable biosensor workflow. 510 Comparing the signal from these samples to corresponding controls without cannabinoids showed clear 511 detection of THC (Fig 9F and G).

512

513 Overall, we developed a portable cannabinoid sensing workflow including a custom-made easy to use 514 device that can be used with a standard cell phone capable of detecting real-life concentrations of 515 cannabinoids from saliva samples. 


\section{DISCUSSION}

520 In this work, we demonstrate the use of GPCRs as the sensing units in yeast-based whole-cell

521 biosensors for specific biotechnological applications. Earlier work established a general framework for 522 integrating heterologous GPCR signaling in yeast ${ }^{5,27}$ and the potential of this technology was highlighted 523 by specific examples including the detection of fungal pathogens ${ }^{24}$ or deorphanization of 524 uncharacterized receptors ${ }^{25}$. However, in order to make GPCR-based biosensors a cost-efficient, 525 sensitive, and widely applicable method, further improvements in this technology were required. Here, 526 we introduce technical improvements and two new reporter modules to achieve sensitivity and dynamic 527 range that enable challenging applications. To highlight the efficiency of the developed system, we 528 selected cannabinoid determination as a key challenge, because requires different biosensor performance characteristics to enable diverse applications.

531 GPCRs are very important as drug targets, and 34\% of FDA approved drugs act on GPCRs ${ }^{43,44}$. CB2, 532 in particular, is the target of multiple ongoing drug discovery efforts ${ }^{45}$. Thus, we start by demonstrating 533 high-throughput screening of synthetic compound libraries using inexpensive and accessible 534 equipment. The modular biosensor design implemented here can be adapted to the study of any other 535 GPCR of interest. Thus, it has the potential to democratize GPCR drug screening, making it available 536 to small research labs or limited budget companies. This is particularly relevant today, as several publicly 537 available repositories for new compounds have been established in the past years ${ }^{46}$. Our biosensors 538 display sensitivity and robustness on par with that of mammalian systems, which are clearly more costly, 
complicated to use, and require specialized equipment, infrastructure, and trained personnel. While

mammalian cells are still indispensable for functional validation, the developed yeast-based biosensor

541 can help narrow down large libraries into a subset of hits that can be further evaluated using mammalian

542 systems. Furthermore, although mammalian cell systems can distinguish off-target effects of drug leads,

543 a yeast-based system can help focus on the effect of a drug directly on the receptor. The low background

544 of the yeast system and its high dynamic range can also be beneficial for detecting more subtle effects

545 of potential pharmacophores that can be subsequently further optimized by chemical functionalization.

547 Subsequently, we address a more complex challenge, by demonstrating the use of the cannabinoid 548 biosensor in bioprospecting. For this application, developing biosensor with high sensitivity and low 549 background is paramount, as the compounds of interest are frequently present in low concentrations 550 and mixed with compounds that can potentially interfere with both the receptor and the reporter. Another 551 challenge is the potential toxicity of target or non-target compounds against the cell line. As a yeast552 based system, our biosensor is less likely to experience toxic effects than animal cells. Using this 553 biosensor, we identified a novel phytocannabinoid, dugelsialactone, a compound that was previously 554 known as an anti-cancer lead ${ }^{47}$, but had never been demonstrated to be a cannabinoid. Interestingly, 555 dugelsialactone showed an $\mathrm{EC}_{50}$ of $2 \mu \mathrm{M}$, exhibiting higher potency than $\mathrm{THC}$.

557 Finally, we demonstrate the development of portable biosensor devices that can easily detect the presence of cannabinoids in real-life samples outside the lab and without the need for expensive equipment or trained personnel. This requires biosensors that are sensitive, robust and with an output that can be measured by eye or common equipment such as a cell phone camera. Contrary to available 
quick tests that are based on antibodies and can only detect a specific cannabinoid, our sensor is able

562 to detect any of the structurally diverse cannabinoids that is a CB2 ligand. This enables quantification

563 of known compounds - characterization of "known-unknown" compounds - or detection of "unknown-

564 unknown" compounds. Examples for such applications include monitoring cannabinoid levels in human

565 urine or saliva samples after therapeutic treatment with cannabinoids, quality/potency control of medical

566 cannabis, or interception of dangerous unknown synthetic cannabinoids. We envision that the portable

567 biosensors can be further matured into commercial devices by optimizing the cell phone accessory

568 devices and software. Currently, the luminescence-based biosensor can generate a measurable signal

569 already after $15 \mathrm{~min}$. Exploiting this speed requires utilization of the full sensitivity of cell phone cameras,

570 improved optics, and additional signal processing. Finally, we foresee that the portable biosensor

571 technology and platform strain can be used to enable detection of numerous other biomarkers using

572 other GPCRs, thus paving the way for smart living diagnostics.

573

574 In addition to the three case studies demonstrated here, our GPCR-based biosensor platform can be

575 expanded to fit numerous other applications. For example, there is a strong potential in using the

576 biosensor in metabolic engineering efforts to monitor and optimize strains for the bioproduction of small

577 molecules or enzymes. Moreover, GPCR-based biosensors can be used to screen mutant libraries for

578 enzymes with improved activity or to drive "directed evolution" of enzymes.

579 


\section{Materials and Methods}

Chemicals and enzymes: All chemicals were reagent grade. CP55940, L-DOPA, O-diadizidine, 5-FOA and the CeLytic $Y$ reagent were purchased from Merck Life Science A/S (Søborg, Denmark). The compounds corresponding to HTS screening hits AGO1 (\#6118258), AGO2 (\#5143116), ANT1 (\#5169183), ANT2 (\#5182514), ANT3 (\#5177027), ANT6 (\#5377528), ANT7 (\#5148134) were obtained from ChemBridge, US. ANT4 (\#3888-1206) and ANT5 (\#3909-7498) were bought from ChemDiv, US. 11-OH-THC and JWH-018 were obtained from LGC Standards Ltd., UK And THC was bought from Chiron AS., Norway. Nano-glo luciferase reagent was bought form Promega, US. The chemical library used for high-throughput screening was obtained from the "Chemical biology and HTS" facility of the University of Copenhagen (https://cbhts.ku.dk/chemical-compound-collection/). Restriction enzymes, and digestion and PCR reaction buffers were from New England Biolabs (NEB), USA. Pfu-X7 DNA Polymerase $^{48}$ was made in- house. MangoMixTM 2x Taq DNA polymerase PCR master mix was purchased from Meridian Life Science Inc., US.

Plant Material: Plant material for the 49 plant species were collected from the Botanical gardens, grown from seeds or collected from the wild; details are given in Table S3. Botanical identification was ensured by MSc. Eduardo Blanco Contreras curator at the Centro de Referencia Botanica de la Universidad Autonoma Agraria Antonio Narro (CREB-UAAAN) where vouchers of the specimens were deposited.

Yeast strain construction: Yeast strains used in this study are listed in Table S1. All strains were produced by genomic integration of DNA fragments (linearized plasmids or PCR products) transformed into the relevant strain either by the lithium acetate method ${ }^{49}$ or by electroporation. Chassis strains were produced by HR based knock-out ${ }^{50}$ and the subsequent strains by HR based modular multi-part integration (Fig S2) (Forman et al., unpublished).

PEG-Lithium acetate mediated transformation: Yeast transformations were performed according to a modified PEG-LiOAc protocol ${ }^{49}$. A $5 \mathrm{~mL}$ saturated overnight YPD culture was diluted to $0.25 \mathrm{OD}$ and grown until $1 \mathrm{OD}$. Then the yeast was pelleted by centrifugation and washed with $0.1 \mathrm{M}$ LiOAc. The cells were then resuspended in $20 \mu \mathrm{L}$ of $0.1 \mathrm{M} \mathrm{LiOAc}$ following by addition of $10 \mu \mathrm{L}$ of heat-denatured salmon sperm DNA and $200 \mu \mathrm{L}$ of PLI solution (45\% Polyethyleneglycol-3350 with 0.1 M LiOAc). For each transformation, $200 \mu \mathrm{L}$ of this cell stock was mixed with linear plasmid DNA to be transformed and heat-shocked at $42^{\circ} \mathrm{C}$ for 30 minutes. Then, the cells were washed with $\mathrm{H}_{2} \mathrm{O}$ and plated on $\mathrm{CM}-\mathrm{U}$ plates.

Electroporation: Yeast cells were inoculated in YPD and grown overnight at $30 \mathrm{C}$ with 150 rpm shaking to a saturated culture. Then, the cells were diluted into $50 \mathrm{~mL}$ of YPD to OD600 $=0.25$ and grown until 
OD600 $=1$. The cells were collected by centrifugation for $5 \mathrm{~min}$ at $4^{\circ} \mathrm{C}$ and $3000 \mathrm{~g}$ and resuspended in $10 \mathrm{~mL}$ ice cold H2O. Next, the cells were treated with EP solution (1M sorbitol, $100 \mathrm{mM}$ LiOAc. $10 \mathrm{mM}$ Hepes ( $\mathrm{pH} 7), 10 \mathrm{mM}$ DTT) for $15 \mathrm{~min}$ at $30^{\circ} \mathrm{C}$. The cells were washed twice with ice cold $1 \mathrm{M}$ sorbitol and resuspended in $0.2 \mathrm{~mL}$ of ice cold $1 \mathrm{M}$ sorbitol. $5 \mu \mathrm{L} \mathrm{PCR}$ product was added to $40 \mu \mathrm{L}$ of yeast cell suspension in a $0.2 \mathrm{~cm}$ electroporation cuvette. The cells were electroporated with a BIO-RAD Gene Pulser Xcell using the standard protocol for S. cerevisiae (25 $\mathrm{F}, 200 \mathrm{ohm}, 1.5 \mathrm{kV})$. The cells were resuspended in $1 \mathrm{~mL} 1 \mathrm{M}$ sorbitol and incubated for $1 \mathrm{~h}$ at $30 \mathrm{C}$. Finally, the cells were collected by centrifugation for $1 \mathrm{~min}$ at $8000 \mathrm{~g}$ at RT, resuspended in $100 \mu \mathrm{L}$ of $1 \mathrm{M}$ sorbitol and plated on selection 624 plates (CM-U).

625

Generation of knock-out mutant strains: Chassis strains were produced by stepwise knocking out STE3, SST2, FAR1, STE12 and GPA1. Briefly, the parent strain was transformed with either knock-out cassette (Table S7) containing the URA3 selectable marker flanked by LoxP sites. This fragment was PCR amplified from the plasmid pUG72 ${ }^{50}$ using primers containing $40-50$ bp overhangs corresponding to the genomic sequence of the KO target gene. The transformed yeast was then plated on yeast selection media (CM-U). Knock-out cassette positive colonies were identified by PCR yeast genotyping using primers YEA85, YEA89 and YEA90 (Table S8). Subsequently a knock-out positive yeast clone was transformed with a plasmid conferring galactose inducible CRE-LOX recombinase expression. Finally, the URA3 selection marker was removed by CRE-LOX recombination and a markerless clone was picked via 5-FOA counter-selection. The procedure was then repeated with the next knock-out. For the GPA1 knock-out, electroporation was used to ensure a high enough transformation rate of the KO cassette. After the last knock-out the CRE-LOX plasmid was removed by curing.

Plasmid construction: Plasmid constructs used in this study are listed in Table S6 All plasmids newly 640 constructed for this study were made with USER cloning ${ }^{51}$.

USER cloning: Promoters, ORFs and other parts (Table S7) were PCR amplified with USER primers restriction enzymes and USER-PCR-fragments were ligated into USER vectors according to Mikkelsen et al., $2012^{52}$.

Primers: All PCR Primers used in this study are listed in Table S8 Primers were ordered from TAG 648 Copenhagen, Denmark. 
650

651

652

653

654

655

656

657

658

659

660

661

662

663

664

665

666

667

668

669

670

671

672

673

674

675

676

677

678

679

680

681

682

683

684

Induction of biosensor strains: Biosensor strains were grown until saturation. Then cells were pelleted by centrifugation and cell density set to OD600 $=5$ (luciferase strains) or 0.5 (betalain or ZsGREEN strains) by resuspending in fresh CM-U media. $20-200 \mu \mathrm{L}$ of cells were dispensed in 96 well plates and inducer added. The cells were then induced for $15 \mathrm{~min}-24 \mathrm{~h}$ at $30 \mathrm{C}$ with $200 \mathrm{rpm}$ shaking. For betalain reporter strains the additives $0.1 \mathrm{mg} / \mathrm{mL}$ Tyrosine, $0.1 \mathrm{mg} / \mathrm{mL}$ L-DOPA, or $0.5 \mathrm{mM}$ o-Diadisidine were added at this stage if relevant.

Fluorescent reporter measurements: The ligand response of the cannabinoid biosensor strains with ZsGreen reporter gene were measured using a Molecular devices SpectraMax-M5 plate reader. $100 \mu \mathrm{L}$ of induced cells were added to black clear-bottom 96-well microplates and the fluorescent signals were read using a $480 \mathrm{~nm}$ excitation, $495 \mathrm{~nm}$ cut-off, and $515 \mathrm{~nm}$ emission wavelength.

Betalain reporter measurements: The signal from the betalain reporter was measured using a Molecular Devices SpectraMax-M5 plate reader with $470 \mathrm{~nm}$ and $520 \mathrm{~nm}$ absorption wavelengths for betaxanthins and betacyanins respectively.

Luciferase reporter assays and measurements: Luciferase signal measurements were made by mixing $25 \mu \mathrm{L}$ of induced cells together with $25 \mu \mathrm{L}$ luciferase reagent (CeLytic $Y$ with $4 \%$ Nano-glo reagent) in a black ProxiPlate ${ }^{\mathrm{TM}}$ Perkin-Elmer (\#6006270). After 10 min incubation luminescence was measured with Molecular Devices SpectraMax-M5 plate reader with $0.5 \mathrm{~s}$ integration time.

Data analysis: Luminescence, fluorescence or colorimetric data was analyzed in OriginPro software. Curve fitting was performed using the sigmoidal fitting "Hill1" or "biHill" algorithm with default settings. LOD was determined as lowest experimental measurement that was significantly different (t-test, $p<$ $0.05)$ from the negative control (no ligand).

High-throughput screening: For each HTS experiment $100 \mu \mathrm{L}$ or $40 \mu \mathrm{L}$ of cells (see "induction of biosensor strains") were dispensed into 96 well or 384 well plates using an OT-2 pipetting robot. Then, $1 \mu \mathrm{L}$ of the chemical library was added to these cells. After incubation (16 h for betanin strain, $3 \mathrm{~h}$ luciferase strain) biosensor output was evaluated by eye (betanin strain) or by mixing in $20 \mu \mathrm{L}$ of developer solution (Celytic $Y$ reagent with 1\% Nano-glo reagent) and luminescence measurement with SpectraMax-M5 plate reader.

Extraction of plant material: Dried plant material was ground to a fine powder using a domestic grinder. Subsequently, powder was suspended in Methanol at a ratio of 1:10 (w/v) and ultrasonicated for $30 \mathrm{~min}$ at room temperature $\left(24^{\circ} \mathrm{C}\right)$ and filtered under reduced pressured. The resulting extracts 
685

686

687

688

689

690

691

692

693

694

695

696

697

698

699

700

701

702

703

704

705

706

707

708

709

710

711

712

713

714

715

716

717

718

719

720

were then dried by rotor evaporation, the dry weights were recorded, and dried crude extracts were resuspended in DMSO to a concentration of $50 \mathrm{mg} / \mathrm{mL}$.

Isolation and identification of compound dugesialactone: The isolation of dugesialactone was performed with a Shimadzu Prominence LC-20A system, consisting of a SIL-10AP autosampler, a LC20AT quaternary pump, a CTO-10ASvp thermostatted column compartment, a SPD-M20A diode array detector detector, and a FRC-10A fraction collector. Around 20 consecutive injections of crude EtOAc extract $(0.2 \mathrm{~mL}$ per injection, $50 \mathrm{mg} / \mathrm{mL}$ in $\mathrm{MeOH})$ were separated at a flow rate of $2 \mathrm{~mL} / \mathrm{min}$ using the above-mentioned solvents with a Phenomenex Luna C18 (2) column $(250 \times 21.2 \mathrm{~mm}, 5 \mu \mathrm{m}, 100 \AA$; Phenomenex, Torrance, CA, USA). Separations were performed using the following elution profile: $0 \mathrm{~min}, 10 \% \mathrm{~B} ; 30 \mathrm{~min}, 100 \% \mathrm{~B} ; 50 \mathrm{~min}, 100 \% \mathrm{~B}$.

NMR experiments: were performed on a $600 \mathrm{MHz}$ Bruker Avance III instrument (operating frequency of $600.13 \mathrm{MHz}$ ) equipped with a cryogenically cooled $1.7-\mathrm{mm} \mathrm{TCl}$ probe head and a Bruker SampleJet sample changer (Bruker Biospin, Karlsruhe, Germany). All experiments were acquired in automation (temperature equilibration to $300 \mathrm{~K}$, optimization of lock parameters, gradient shimming, and setting of receiver gain). ${ }^{1} \mathrm{H}$ NMR spectra were acquired with $30^{\circ}$-pulses and $64 \mathrm{k}$ data points. $2 \mathrm{D}$ homo- and heteronuclear experiments were acquired with 2048 data points in the direct dimension and 128 (HMBC) or 512 (DQF-COSY) or 256 (multiplicity edited HSQC and NOESY) data points in the indirect dimension. IconNMR ver. 4.2 (Bruker Biospin, Karlsruhe, Germany) was used for controlling automated sample change and acquisition of NMR data, whereas Topspin ver. 3.5 (Bruker Biospin, Karlsruhe, Germany) was used for acquisition and processing of NMR data.

Portable biosensor experiments: Portable biosensor devices (Fig. 8 and 9) were constructed by Flemming Frederiksen and team at the Workshops, Department of Plant and Environmental Sciences, University of Copenhagen. Betalain portable biosensor experiments were performed by dispensing 0.5 $\mathrm{mL}$ of KM205 biosensor strain culture (see "induction of biosensor strains") in each well (Fig. 8) and adding $5 \mu \mathrm{L}$ of $100 \mathrm{x}$ inducer. The devices were covered with a plastic film and incubated for $16 \mathrm{~h}$ at $30^{\circ} \mathrm{C}$ with $200 \mathrm{rpm}$ shaking. After incubation the devices were visualized by eye and photographed. Betalain reporter output was quantified using a cell phone camera by calculating the "redness" of each sample as previously described by Ostrov et al., $2017^{24}$.

Luciferase portable biosensor experiments were performed by dispensing 37.5 or $50 \mu \mathrm{L}$ of KM205 biosensor strain culture (see "induction of biosensor strains") in each well (Fig. 9) together with 1 ul of $100 \mathrm{x}$ inducer or $12.5 \mu \mathrm{L}$ of "artificial saliva with mucin" (Pickering \#1700-03169) spiked with either cannabinoid and incubating for $3 \mathrm{~h}$ at RT. After incubation $50 \mu \mathrm{L}$ of developer reagent (Celytic $Y$ reagen with $4 \%$ Nano-glo reagent) was added to each well and incubated $10 \mathrm{~min}$ at RT. Then, the device was 
assembled by adding the adaptor part (Fig. 9), macro lens (Xenvo Clarus 15x) and attaching the assembly a Huawei Mate 30 pro cell phone. RAW format photographs were taken in "pro" mode using 1 s or 30 s exposure time, 1000 or 6400 ISO setting, EV=0, auto focus-F, and auto white balance settings. Since the peak wavelength of NanoLuc $(450 \mathrm{~nm})$ is the same as that of a typical bayer filter, Intensity of luminescence was quantified from the RAW picture by measuring intensity of the blue channel only. The RAW pictures were analyzed with the RawTherapee 5.8 open-source software. To isolate the blue channel no demosaicing was chosen, and the red and green channel were disabled. Blue intensity was measured with the analysis tool.

729

\section{Acknowledgements}

We would like to thank Dr. Renaud Wagner (Institut de Recherche de l'Ecole de Biotechnologie de Strasbourg) for providing the CB2 clone, Flemming Frederiksen and team from construction of portable biosensor devices, and Marek Miettinen for the assistance with portable biosensor sensor imaging and analysis development. We would also like to thank Dr. Simon Dusséaux (University of Copenhagen, Denmark) for critically reading the manuscript. The project leading to this application has received funding from the European Union's Horizon 2020 research and innovation programme under the Marie Sklodowska-Curie grant agreement No. 845835. This work was also supported by the Novo Nordisk Foundation grants NNF16OC0021760 and NNF190C0055204 (to SCK), NNF17OC0027646 (to SB), NNF18OC0031872 (to KM), and NNF16OC0021616 (to DS).

\section{Author contributions:}

$744 \mathrm{KM}$ : Conceived the project, designed and coordinated research, constructed yeast strains, performed 745 biosensor experiments, analyzed data, and wrote the manuscript.

$746 \mathrm{NL}$ : Constructed yeast strains, performed biosensor experiments, analyzed data

747 LRH: Constructed yeast strains, performed biosensor experiments, analyzed data

748 AAR: Sourced and prepared plant material, performed biosensor experiments, performed

749 chromatography, analyzed data

750 YZ: Performed compound purification, performed chromatography, NMR data analysis and structure 751 elucidation.

752 IEN: Performed biosensor experiments, analyzed data

753 DS: NMR data analysis and structure elucidation.

754 SB: Designed research.

755 SCK: Conceived the project, designed and coordinated research, and wrote the manuscript. 
757 Competing financial interests

758 The authors declare no competing financial interest.

759

760 Supplementary Materials

761 Supplementary Information containing: Supplementary Tables $1-9$ and Supplementary 762 Figures $1-13$.

763

764

765

766

767

768

769

770

771 
1 Lagerstrom, M. C. \& Schioth, H. B. Nat Rev Drug Discov 7, 339-357, doi:10.1038/nrd2518 (2008).

$2 \quad$ Brown, A. J. et al. J Pharmacol Exp Ther 337, 236-246, doi:10.1124/jpet.110.172650 (2011).

3 Brown, A. J. et al. Yeast 16, 11-22, doi:10.1002/(SICI)1097-0061(20000115)16:1<11::AIDYEA502>3.0.CO;2-K (2000).

4 Dowell, S. J. \& Brown, A. J. Methods Mol Biol 552, 213-229, doi:10.1007/978-1-60327-317-6_15 (2009).

$5 \quad$ Shaw, W. M. et al. Cell 177, 782-796 e727, doi:10.1016/j.cell.2019.02.023 (2019).

$6 \quad$ Ren, M. et al. Sci Adv 5, eaaw1391, doi:10.1126/sciadv.aaw1391 (2019).

7

8

Riva, N. et al. Lancet Neurol 18, 155-164, doi:10.1016/S1474-4422(18)30406-X (2019).

de Lago, E., Moreno-Martet, M., Cabranes, A., Ramos, J. A. \& Fernandez-Ruiz, J. Neuropharmacology 62, 2299-2308, doi:10.1016/j.neuropharm.2012.01.030 (2012).

Financialnewsmedia.com. Global Market for Cannabinoid-Based Pharmaceuticals Expected to Reach $\$ 50$ Billion by 2029, <https://www.prnewswire.com/news-releases/global-market-forcannabinoid-based-pharmaceuticals-expected-to-reach-50-billion-by-2029-301185483.html> (2020).

Matsuda, L. A., Lolait, S. J., Brownstein, M. J., Young, A. C. \& Bonner, T. I. Nature 346, 561564, doi:10.1038/346561a0 (1990).

11 Munro, S., Thomas, K. L. \& Abu-Shaar, M. Nature 365, 61-65, doi:10.1038/365061a0 (1993).

12 Shahbazi, F., Grandi, V., Banerjee, A. \& Trant, J. F. iScience 23, 101301, doi:10.1016/j.isci.2020.101301 (2020).

13 Cristino, L., Bisogno, T. \& Di Marzo, V. Nat Rev Neurol 16, 9-29, doi:10.1038/s41582-019-0284z (2020).

14 Chicca, A. et al. Sci Adv 4, eaat2166, doi:10.1126/sciadv.aat2166 (2018).

Walsh, K. B. \& Andersen, H. K. Int J Mol Sci 21, doi:10.3390/ijms21176115 (2020).

Peacock, A. et al. Lancet 394, 1668-1684, doi:10.1016/S0140-6736(19)32231-7 (2019).

Van Sickle, M. D. et al. Science 310, 329-332, doi:10.1126/science.1115740 (2005).

Cabral, G. A., Raborn, E. S., Griffin, L., Dennis, J. \& Marciano-Cabral, F. Br J Pharmacol 153, 240-251, doi:10.1038/sj.bjp.0707584 (2008).

9 Atwood, B. K. \& Mackie, K. Br J Pharmacol 160, 467-479, doi:10.1111/j.14765381.2010.00729.x (2010).

Stempel, A. V. et al. Neuron 90, 795-809, doi:10.1016/j.neuron.2016.03.034 (2016).

Xiang, W. et al. Nat Commun 9, 2574, doi:10.1038/s41467-018-04999-8 (2018).

Adams, A. J. et al. N Engl J Med 376, 235-242, doi:10.1056/NEJMoa1610300 (2017).

Normile, D. Synthetic cannabis deaths sound alarms in Australia, $<$ https://www.sciencemag.org/news/2015/01/synthetic-cannabis-deaths-sound-alarmsaustralia> (2015).

Ostrov, N. et al. Sci Adv 3, e1603221, doi:10.1126/sciadv.1603221 (2017).

Yasi, E. A. et al. Biochemistry 58, 2160-2166, doi:10.1021/acs.biochem.8b01208 (2019).

Yasi, E. A., Allen, A. A., Sugianto, W. \& Peralta-Yahya, P. ACS Synth Biol 8, 2710-2717, doi:10.1021/acssynbio.9b00310 (2019).

28 Scott, B. M. et al. SLAS Discov 24, 969-977, doi:10.1177/2472555219875934 (2019).

29

Ehrenworth, A. M., Claiborne, T. \& Peralta-Yahya, P. Biochemistry 56, 5471-5475, doi:10.1021/acs.biochem.7b00605 (2017).

Soethoudt, M. et al. Nat Commun 8, 13958, doi:10.1038/ncomms13958 (2017).

Bush, A. et al. Mol Syst Biol 12, 898, doi:10.15252/msb.20166910 (2016).

Nakamura, Y., Ishii, J. \& Kondo, A. PLoS One 8, e82237, doi:10.1371/journal.pone.0082237 (2013). 


\begin{tabular}{|c|c|c|}
\hline $\begin{array}{l}24 \\
25\end{array}$ & 34 & $\begin{array}{l}\text { Lee, M. E., DeLoache, W. C., Cervantes, B. \& Dueber, J. E. ACS Synth Biol 4, 975-986, } \\
\text { doi:10.1021/sb500366v (2015). }\end{array}$ \\
\hline & 35 & Roberts, C. J. et al. Science 287, 873-880, doi:10.1126/science.287.5454.873 (2000). \\
\hline 27 & 36 & O'Malley, M. A. et al. Protein Sci 18, 2356-2370, doi:10.1002/pro.246 (2009). \\
\hline $\begin{array}{l}28 \\
29\end{array}$ & 37 & $\begin{array}{l}\text { Cid, V. J., Alvarez, A. M., Santos, A. I., Nombela, C. \& Sanchez, M. Yeast 10, 747-756, } \\
\text { doi:10.1002/yea.320100606 (1994). }\end{array}$ \\
\hline 30 & 38 & $\begin{array}{l}\text { Grewal, P. S., Modavi, C., Russ, Z. N., Harris, N. C. \& Dueber, J. E. Metab Eng 45, 180-188, } \\
\text { doi:10.1016/j.ymben.2017.12.008 (2018). }\end{array}$ \\
\hline 32 & 39 & Hall, M. P. et al. ACS Chem Biol 7, 1848-1857, doi:10.1021/cb3002478 (2012). \\
\hline & 40 & Bohlmann, F. \& Zdero, C. Chemische Berichte 109, 1436-1445 (1976). \\
\hline & 41 & $\begin{array}{l}\text { Huo, Y., Shi, H., Li, W., Wang, M. \& Li, X. J Pharm Biomed Anal 51, 942-946, } \\
\text { doi:10.1016/j.jpba.2009.09.032 (2010). }\end{array}$ \\
\hline 37 & 42 & $\begin{array}{l}\text { Milman, G., Schwope, D. M., Gorelick, D. A. \& Huestis, M. A. Clin Chim Acta 413, 765-770, } \\
\text { doi:10.1016/j.cca.2012.01.011 (2012). }\end{array}$ \\
\hline 38 & 43 & P. A. Mol Pharmacol 93, 251-258, doi:10.1124/mol.117.111062 (2018). \\
\hline & 44 & $\begin{array}{l}\text { Andersen, M., Schioth, H. B. \& Gloriam, D. E. Nat Rev Drug } \\
2017.178 \text { (2017). }\end{array}$ \\
\hline 41 & 45 & Brennecke, B. et al. Pharm Pat Anal 10, 111-163, doi:10.4155/ppa-2021-0002 (2021). \\
\hline 4 & 46 & Nami, F. https://www.dk-openscreen.dk/, <https://www.dk-openscreen.dk/> (2021). \\
\hline 4 & 47 & Calera, M. R. et al. Phytochemistry 40, 419-425, doi:10.1016/0031-9422(95)00257-8 (1995). \\
\hline & 48 & Norholm, M. H. BMC Biotechnol 10, 21, doi:10.1186/1472-6750-10-21 (2010). \\
\hline & 49 & Gietz, R. D. \& Schiestl, R. H. Nat Protoc 2, 31-34, doi:10.1038/nprot.2007.13 (2007). \\
\hline 46 & 50 & $\begin{array}{l}\text { Gueldener, U., Heinisch, J., Koehler, G. J., Voss, D. \& Hegemann, J. H. Nucleic Acids Res 30, } \\
\text { e23, doi:10.1093/nar/30.6.e23 (2002). }\end{array}$ \\
\hline 848 & 51 & $\begin{array}{l}\text { Nour-Eldin, H. H., Geu-Flores, F. \& Halkier, B. A. Methods Mol Biol 643, 185-200, } \\
\text { doi:10.1007/978-1-60761-723-5_13 (2010). }\end{array}$ \\
\hline & & h Fāa 14 104-111 doi.10 1016/i vmhen 201201006 (2012) \\
\hline
\end{tabular}




\section{Supplementary Files}

This is a list of supplementary files associated with this preprint. Click to download.

- Supplementallnformationv8.pdf

- nrreportingsummaryNCOMMS2128728.pdf 